\title{
A Network-Based Approach to Deciphering A Dynamic Microbiome's Response to A Subtle Perturbation
}

Grace Tzun-Wen Shaw

Academia Sinica https://orcid.org/0000-0003-4393-3655

An-Chi Liu ( $\sim$ d00631001@ntu.edu.tw )

National Taiwan University

Chieh-Yin Weng

Academia Sinica

Yi-Chun Chen

National Taiwan University

Cheng-Yu Chen

Academia Sinica

Francis Cheng-Hsuan Weng

Academia Sinica

Daryi Wang

Academia Sinica

Chu-Yang Chou ( $\nabla$ choucy@ntu.edu.tw)

https://orcid.org/0000-0003-0861-5520

\section{Research}

Keywords: Bioaugmentation, Anaerobic digestion, Network topology, Biodiversity, Subtle perturbation, MetaMIS, Microbial interactions, Motif, Co-occurrence pattern

Posted Date: May 14th, 2020

DOl: https://doi.org/10.21203/rs.3.rs-27654/v1

License: (c) (i) This work is licensed under a Creative Commons Attribution 4.0 International License. Read Full License 


\section{Abstract}

Background: Over the past decades, one main issue that has emerged in ecological and environmental research is how losses in biodiversity influence ecosystem dynamics and functioning, and consequently human society. Declines in biodiversity lead to more than just species loss. The exponentially growing human population and its exploitation of natural resources affect the environment from local to global scales via climate change, global warming, and other human-driven environmental problems. Although biodiversity is a common indicator of ecosystem functioning, it is difficult to measure biodiversity in microbial communities exposed to moderate or chronic environmental perturbations. Consequently, there is a need for alternative bioindicators to detect, measure, and monitor gradual changes in microbial communities against these slight, chronic, and continuous perturbations.

Results: Recent studies have proven that co-occurrence patterns are effective for measuring responses to subtle perturbations. In this study, we mimicked dynamic microbial ecosystems under pressure from subtle perturbations by adding a key microbial species and comparing network topologies. The present study is a comprehensive investigation of how a network-oriented method can be used to track microbiome dynamics after a key species, $S$. acidaminiphila, is added into anaerobic digestion reactors of swine manure. Microbial networks before and after bioaugmentation with $S$. acidaminiphila showed diverse topological niches and 4-node motifs in which microbes with co-occurrence patterns played the central roles in regulating and adjusting the intertwined relationships among microorganisms in response to the subtle environmental changes.

Conclusions: This study demonstrates that microbial networks are a good bioindicator for chronic perturbation and should be applied in a variety of ecological investigations.

\section{Background:}

The past decades have seen remarkable progress in understanding how human activities influence Earth's ecosystems [1]. The loss of biological diversity-in terms of all life on earth, genetic variation among creatures, and entire ecosystems-impacts ecological processes, ecosystem services, human society, and economics. Most recently, the intertwined relationship between global environmental change and biodiversity dynamics has become a new impetus for ecological research [2,3]. Major strides have been made in rethinking how we conserve natural resources sustainably, redefining how we evaluate the quality of ecosystems, and refining biological indicators to accurately measure biodiversity $[1,2,4]$. A powerful bioindicator-e.g. plants, planktons, animals, and microbes-can show how quickly a natural surrounding is changing, or predict how it will change. For example, marine pollution can be detected by measuring changes in phytoplankton diversity $[5,6]$. The quality of aquatic and terrestrial habitats can be monitored by changes in rotifer [7], leech [6], and macrobenthos [8] populations, among others. Several studies recently highlighted the importance of microorganisms for determining low levels of contaminants and small biological changes, owing to their rapid growth $[9,10]$. 
It is easier to measure ecosystem functioning using microbial biodiversity than animal or plant biodiversity. Current negative trends in microbial biodiversity are mainly due to climbing anthropogenic pressures, e.g. resource consumption, invasive alien species, pollution, and habitat destruction, and rapid climate change $[1,4]$. These large perturbations directly affect microbial species, leading to compositional changes, and indirectly alter species' behaviors and the strength of inter-species interactions, all of which result in a dynamic ecological network. However, most conventional indexes capturing taxonomic diversity based only on species abundance, richness, and evenness-such as the Shannon index [11], Simpson index [12], and Chao-1 index [13]-do not assess the effect of interrelatedness among species on the stability of the entire ecosystem, and therefore lack the sensitivity to respond to subtle and chronic environmental degradation.

Microbes interact with their communities in a complicated way. Using correlation, or co-occurrence or coexclusion, to measure microbial relationships [14] is the simplest approach to potentially identifying pairs that are metabolically complementary [15]. Mutualistic microbes may benefit each other and correlate positively among samples. Competitive microbes may compete with one another, leading to a negative correlation trend $[14,15]$. Studies of co-occurrence networks in microbial communities have confirmed the connection between network structure and chronic (and weak) environmental changes due to soil anthropization [16], litter quality [17], and air pollution [18, 19], in which microbial biodiversity-e.g. Shannon and inverse Simpson index-might therefore lead to the conclusion that the ecosystem remains unaltered if the perturbation is of moderate intensity.

Over the past decades, researchers have become increasingly aware of subtle changes in environmental conditions during studies on ecological sustainability and the impacts of anthropogenic and natural processes. A robust microbial ecosystem has four major drivers influencing microbial biodiversity, which can be informative or sensitive indicators of an ecosystem's response to subtle perturbations: rare species effect, resistance/resilience effect, spatial effect, and interactive effect [20]. Measuring microbial diversity at different spatial and temporal scales is another way of using microorganisms as indicators. Priority effects during microbial colonization have long-lasting consequences for the development of microbial communities and constitutes a major barrier to entry for microbes entering a community; this is also called colonization resistance [21, 22].

The purpose of this study is to describe the application of microbial networks to detect a subtle and chronic environmental change. More specifically, this study was undertaken to understand how a subtle perturbation can illustrate the concept of colonization resistance using a novel network-based bioaugmentation approach in an anaerobic digestion system, and to suggest some practical implications and connections of co-occurrence patterns to future work. In this paper, we present a conceptual framework that links subtle perturbations; the unchangeableness of biodiversity; and the dynamics of microbial composition, co-occurrence, and networks. Their unique capabilities make the use of microbial networks to detect subtle perturbations much more useful than conventional biodiversity measurements.

\section{Materials And Methods:}




\section{Bench-top anaerobic digesters}

A set of ten 5-liter bench-top continuous stirred tank reactors (CSTRs) with a working volume of $3 \mathrm{~L}$ was used in series or in parallel [23]. The CSTR design can completely homogenize the digester content and collect samples in a straightforward manner. The primary feedstock for these digesters was pig manure collected from pig farms in DaXi, ZaoQiao Township, Miaoli County, Taiwan (we received approval from the farm owners to collect concentrated swine manures). These anaerobic digesters were operated under mesophilic $\left(37^{\circ} \mathrm{C}\right)$ conditions using a hydraulic retention time (HRT) of 10 days, $8 \%$ total solids (TS), and a stirring speed of $60 \mathrm{rpm}$ without $\mathrm{pH}$ control. All anaerobic digesters were initiated using $1.2 \mathrm{~L}$ of inoculum from the anaerobic digesters, which were maintained by Dr. Chu-Yang Chou's laboratory in the Department of Biomechatronics Engineering, National Taiwan University (Taipei, Taiwan), and $1.8 \mathrm{~L}$ of swine manure from the pig farms mentioned above.

\section{Isolation of pure culture from anaerobic digesters}

Four kinds of media were used to isolate aerobic bacteria: LB (Luria-Bertani) (Difico), TSB (tryptic soy broth) (Bacto), NB (nutrient broth) (Difico), and R2A (0.5 g Bacto protesost peptone, $0.5 \mathrm{~g}$ Bacto casamino acids, $0.5 \mathrm{~g}$ Bacto dextrose, $0.5 \mathrm{~g}$ soluble starch, potassium phosphate, $0.3 \mathrm{~g}$ didbasic, $0.5 \mathrm{~g}$ Bacto yeast extract, $0.05 \mathrm{~g}$ magnesium sulfate, $0.3 \mathrm{~g}$ sodium pyruvate). All four were solidified by adding $1.5 \%$ agar. The media were autoclaved for $20 \mathrm{~min}$ at $121^{\circ} \mathrm{C}$. Then, an effluent sludge sample from mesophilic $\left(37^{\circ} \mathrm{C}\right.$ ) anaerobic digesters underwent serial dilution $\left(10^{-1}-10^{-7}\right) .100 \mu \mathrm{L}$ of the $10^{-4}-10^{-7}$ dilution samples was added to the $900 \mu \mathrm{L}$ media and spread using the streak-plate method with glass bead cylinders. All media with sludge samples were incubated at $37^{\circ} \mathrm{C}$ for 1 to 7 days for single colonies to emerge on the new plate and be used for pure culture isolation.

Pure cultures were preserved for the subsequent experiments by enriching in the medium at $37^{\circ} \mathrm{C}$ in a horizontal shaker at $200 \mathrm{rpm}$. When the culture reached the log phase or stationary phase, $0.6 \mathrm{~mL}$ was maintained in a $1.5 \mathrm{~mL}$ tube with $0.4 \mathrm{~mL} 80 \%$ (W/V) glycerol; the glycerol concentration reached $20 \%$ (W/V). Finally, the culture was mixed well and stored at $-20^{\circ} \mathrm{C}$ or $-80^{\circ} \mathrm{C}$.

\section{Biochemical methane potential (BMP) tests}

BMP is a simple and inexpensive bioassay that measures relative biodegradability by monitoring cumulative methane production in an anaerobic digestion system [24]. The conventional BMP test typically takes two weeks when the amount of biogas or methane remains the same. This study, however, adopted a simplified three-day BMP test to quickly examine biogas and methane yields for each selected key microbial candidate. The batch tests were carried out using $1 \mathrm{~L}$ serum bottles (Schott Glaswerke); $450 \mathrm{~mL}$ effluents from mesophilic anaerobic digesters and $50 \mathrm{~mL}$ swine manure with $8 \%$ TS were used, different bacterial cell counts ranging from $10^{3}$ to $10^{8}$ were added, flushed with nitrogen at the beginning of the assay, and then placed in a thermostatic water bath at a mesophilic temperature $\left(37^{\circ} \mathrm{C}\right)$. All bottles were connected to a water trap and a gas bag to collect biogas. Biogas production and methane content were measured once daily until no more biogas production observed. 


\section{Bioaugmentation on CSTR}

After the steady state was achieved-chemical oxygen demand (COD) became constant or the production of biogas or methane became invariant $-1 \mathrm{~mL}$ of a bioaugmented species, Stenotrophomonas acidaminiphila $\left(7.2 \times 10^{9} \mathrm{CFU} / \mathrm{mL}\right)$, was added to five reactors daily, and another five reactors were maintained as before. All manure effluents $(300 \mathrm{~mL})$ were collected daily and kept frozen in a $-20^{\circ} \mathrm{C}$ freezer and thawed before use. To avoid air leakage into the digester, a peristaltic pump (Masterflex model No. 7553-80, Cole-Parmer Instrument Co., IL., USA) was connected during the feeding and sampling processes. The $\mathrm{pH}, \mathrm{TS}$, and COD (colorimetric method) of the influent and effluent samples were analyzed according to standard methods [25]. Total gas or biogas production was quantified by a wet test gas meter (W-NK-0.5, Shinagawa Co., Tokyo, Japan). The methane composition was measured by a gas chromatograph (GC-8700T, China Chromatography Co., Taiwan) equipped with a thermal conductivity detector (GC-TCD) and a Porapak Q column with helium as the carrier gas, and calibrated with a gas standard consisting of $100 \%$ methane.

\section{DNA extraction}

To isolate bacterial and archaeal DNA from collected samples, all DNA extractions were performed with the PowerSoil ${ }^{\circledR}$ DNA Isolation Kit (Mo Bio Laboratories, USA) according to the manufacturer's instructions. The quality of DNA extracts was checked by a Nanodrop-1000 Spectrophotometer (Thermo Scientific, Wilmington, DE, USA). The amount of DNA was determined using a Quant-iT dsDNA HS assay kit and a Qubit fluorometer (Invitrogen, Life Technologies, Carlsbad, CA., USA). All above-mentioned procedures were performed in a laminar flow cabinet to avoid contamination.

\section{PCR amplification and 16S rRNA sequencing}

Purified DNA extracts were amplified using the primers of a modified 341F (CCTAYGGGRBGCASCAG) and a modified 806R (GGACTACNNGGGTATCTAAT) [25] fused with Illumina overhanging adapters, which amplified a DNA fragment of about $533 \mathrm{bp}$ containing the V3 and V4 hypervariable regions of the $16 \mathrm{~S}$ rRNA gene [26]. PCR amplification was performed in a $30 \mu \mathrm{L}$ reaction volume using $2 X$ Phusion Flash High-Fidelity PCR Master Mix (Finnzymes Oy, Finland), with the following incubation conditions: $98^{\circ} \mathrm{C}$ for $3 \mathrm{~min}$; followed by 25 cycles of $98^{\circ} \mathrm{C}$ for $30 \mathrm{~s}, 56^{\circ} \mathrm{C}$ for $30 \mathrm{~s}$, and $72{ }^{\circ} \mathrm{C}$ for $30 \mathrm{~s}$; and a final extension of $72{ }^{\circ} \mathrm{C}$ for $5 \mathrm{~min}$. After PCR, the DNA products were confirmed by $2 \%$ agarose gel electrophoresis with Trisacetate-EDTA (TAE) buffer (TOOLS Biotechnology Co., Ltd. Taiwan) and purified by NucleoSpin® Gel and PCR Clean-up (Macherey-Nagel GmbH \& Co. KG, Düren, Germany). After the quality of all cleaned PCR products was confirmed by the Nanodrop-1000 Spectrophotometer (Thermo Scientific, Wilmington, DE, USA), the purified amplicons were processed using the Illumina standard protocol for 16S rRNA sequencing library preparation, and sequenced by the MiSeq platform with the reagent kit v3.

\section{Sequence processing}

For paired-end sequencing of 16S-rRNA gene amplicons, all sequences in the FASTQ format were merged using FLASH [27] with standard parameters except for the maximum overlap parameter, which was set to 
150. The filtering process of merged reads was manipulated by MOTHUR [28]. Primers and low-quality sequences-fewer than 375 bases long, with homopolymers longer than eight nucleotides, or with a quality score $<30-$ were removed using trim.seqs. After the trimming step, nonredundant sequences were generated by the unique.seqs command, then clustered by a criterion of a $97 \%$ sequence similarity using UPARSE [29], and chimeric sequences were eliminated with the OTU-picking step. The classify.seqs command classified sequences into different taxonomies based on the RDP classifier with rainset14_032015.rdp and a confidence score threshold of 80\% in MOTHUR [28]. Sequences assigned to Chloroplast, Mitochondria, Eukaryota, or an unknown kingdom were discarded. 16S rRNA gene copy number (GCN) was adjusted on taxon abundance [30] to generate an OTU table. All sequenced samples were deposited in the NCBI Short Read Archive under BioProject PRJNA629428 (SRR11649175 SRR11649188).

\section{Statistical analysis and classification of microbial members}

For each microbial abundance table at the different taxonomic levels, a relative abundance, $x_{i k}$ was derived from $i=1, \ldots, N$ microbial members and $k=1, \ldots, t$ time-series samples under a specific condition, e.g. with (B) or without bioaugmentation (C). The averaged abundance level of a microbial member among all samples was calculated as:

$$
\bar{x}_{i}=\frac{\sum_{k=1}^{t}\left(x_{i k}\right)}{N\left(x_{i k}>0\right)}
$$

1

Therefore, a microbial member could be defined as having a high or rare abundance level, while the average of abundance levels was larger than $1 \%$ or smaller than $0.1 \%$ of the total number of sequences. Microbes with a mean relative abundance from 0.1 to $1 \%$ were classified as the low abundance level group. Finally, a core microbial member was defined as a microbe conveying relative abundances among all time-series samples.

A paired-samples Student's t test was performed for samples collected on the same date for the control and bioaugmentation group to identify differences in microbial abundance. Correlated abundance patterns for microbes were measured using the formulas below, where $\mathrm{C}$ and $\mathrm{B}$ indicate the control and bioaugmentation, respectively.

$$
\mathrm{r}_{\mathrm{C}_{\mathrm{i}} \mathrm{B}_{\mathrm{i}}}=\frac{\sum_{k=1}^{t}\left(x_{i k}^{C}-\bar{x}_{i}^{C}\right)\left(x_{i k}^{B}-\bar{x}_{i}^{B}\right)}{\sqrt{\sum_{k=1}^{t}\left(x_{i k}^{C}-\bar{x}_{i}^{C}\right)^{2}} \sqrt{\sum_{k=1}^{t}\left(x_{i k}^{B}-\bar{x}_{i}^{B}\right)^{2}}}
$$




$$
\mathrm{r}_{\mathrm{C}_{\mathrm{i}} \mathrm{C}_{\mathrm{j}}}=\frac{\sum_{k=1}^{t}\left(x_{i k}^{C}-\bar{x}_{i}^{C}\right)\left(x_{j k}^{C}-\bar{x}_{j}^{C}\right)}{\sqrt{\sum_{k=1}^{t}\left(x_{i k}^{C}-\bar{x}_{i}^{C}\right)^{2}} \sqrt{\sum_{k=1}^{t}\left(x_{j k}^{C}-\bar{x}_{j}^{C}\right)^{2}}}
$$

$\mathrm{r}_{\mathrm{C}_{\mathrm{i}} \mathrm{B}_{\mathrm{i}}}$ calculated the Pearson correlation for abundance profiles of a microbe before and after bioaugmentation. $\mathrm{r}_{\mathrm{C}_{\mathrm{i}} \mathrm{C}_{\mathrm{j}}}$ measured Pearson correlation between two different microbes under the same conditions. The aim of $\mathrm{r}_{\mathrm{C}_{\mathrm{i}} \mathrm{B}_{\mathrm{i}}}$ was to identify microbes with conserved abundance patterns under the control and bioaugmentation treatments. Under the same condition (control or bioaugmentation), $\mathrm{r}_{\mathrm{C}_{\mathrm{i}} \mathrm{C}_{\mathrm{j}}}$ or $\mathrm{r}_{\mathrm{B}_{\mathrm{i}} \mathrm{B}_{\mathrm{j}}}$ was used to capture microbes with similar abundance patterns to microbes with significant $\mathrm{r}_{\mathrm{C}_{\mathrm{i}} \mathrm{B}_{\mathrm{i}}}$. All statistical significance levels in this study were set to 0.05 .

To understand how microbes with co-occurrence patterns, $\mathrm{r}_{\mathrm{C}_{\mathrm{i}} \mathrm{B}_{\mathrm{i}}}, \mathrm{r}_{\mathrm{C}_{\mathrm{i}} \mathrm{C}_{\mathrm{j}}}$, and $\mathrm{r}_{\mathrm{B}_{\mathrm{i}} \mathrm{B}_{\mathrm{j}}}$ influenced the entire microbiome, co-occurrence clusters were determined using the following steps. First, two abundance profiles from the control and bioaugmentation treatments were combined and the clustering process was performed using the average linkage method with Pearson correlation, which was conducted on the generalized association plots (GAP) software [31]. Second, the abundance correlations $\left(\mathrm{r}_{\mathrm{C}_{\mathrm{i}} \mathrm{C}_{\mathrm{j}}}\right.$ or $\left.\mathrm{r}_{\mathrm{B}_{\mathrm{i}} \mathrm{B}_{\mathrm{j}}}\right)$ of microbes in the same co-occurrence cluster were tested for statistical significance, and one of the paired microbes should have been identified as having a statistically significant $\mathrm{r}_{\mathrm{C}_{\mathrm{i}} \mathrm{B}_{\mathrm{i}}}$. Microbes with significant correlations in $\mathrm{r}_{\mathrm{C}_{\mathrm{i}} \mathrm{C}_{\mathrm{j}}}$ or $\mathrm{r}_{\mathrm{B}_{\mathrm{i}} \mathrm{B}_{\mathrm{j}}}$ were retained in the cluster. Finally, microbes with significant $\mathrm{r}_{\mathrm{C}_{\mathrm{i}} \mathrm{C}_{\mathrm{j}}}\left(\right.$ or $\left.\mathrm{r}_{\mathrm{B}_{\mathrm{i}} \mathrm{B}_{\mathrm{j}}}\right)$ were classified as having a conserved abundance pattern before (or after) bioaugmentation. The abundance patterns of these microbes should be heavily influenced to one of the microbes with significant $\mathrm{r}_{\mathrm{C}_{\mathrm{i}} \mathrm{B}_{\mathrm{i}}}$.

\section{Inference in the microbial interaction network}

To infer microbial interactions, a simulation prediction process based on the Lotka-Volterra model was performed by MetaMIS [32]. For each condition (control or bioaugmentation), we generated 1000 microbial networks from the microbial abundance profile with $\mathrm{N}$ microbial members and $\mathrm{T}$ time-series samples. Each microbial network was randomly predicted by $90 \%$ of $\mathrm{N}$ microbial members. Then, microbial interactions with $90 \%$ coherent interactive outcomes among all predicted interactions were considered reliable after proportional tests were performed; they were used in the subsequent analysis and to construct a microbial network.

\section{Topological niche of microbial networks}

Three topological measurements-indegree, betweenness, and eigenvector centralities-were conducted using Gephi software [33] for each microbial network, in which a node indicated a microbial member $i$ and an edge represented the interactive relation between two microbes. Indegree centrality measured how many other microbes influenced a target microbe and was indicated by node color. A larger indegree 
centrality (darker red) implies that this microbe was influenced by more microbes or interactions. In a microbial network, two microbes must have more than one path. Therefore, betweenness centrality of a microbe is the number of these shortest paths that passed this microbe, and was represented by node size. A larger node indicates a microbe with a larger betweenness centrality. Eigenvector centrality measures the importance of a microbe in a network based on its connections; a microbe was deemed important if it was easy to link it to other influential microbes and it was located in the center of the network.

To systematically identify microbes with a topological niche between two microbial networks ( $B$ and $C$ ), indegree and betweenness centralities at different interactive strengths were calculated and compared for tow microbial networks.

$$
\text { Niche }_{i}^{\text {Centrality }}=\frac{\sum_{S=\text { top500 }}^{\text {Allinteractions }}\left(\text { Centrality }{ }_{i, S}^{B}-\text { Centrality }_{i, S}^{C}\right)}{\max \left\{\left[\text { Centrality } i_{i, S}^{B}-\text { Centrality } y_{i, S}^{C}\right]_{S=\text { top } 500}^{\text {Allinteractions }}\right\}}
$$

Thus, there were two types of Niche $e_{i}^{\text {Centrality }}-N i c h e_{i}^{\text {Indegree }}$ and $N i c h e_{i}^{\text {Betweenness }}$-and it ranged from -1 and 1. If a microbe's Niche $e_{i}^{\text {Centrality }}$ was -0.1 to 0.1 , it was considered as non-influential topological niche or there was no difference between the control and bioaugmentation treatments. If a microbe's $N i c h e_{i}^{\text {Indegree }}$ and $N i c h e_{i}^{\text {Betweenness }}$ were $>0.1$, it was identified as having a topological niche after bioaugmentation. When both $N i c h e_{i}^{\text {Indegree }}$ and $N i c h e_{i}^{\text {Betweenness }}$ were $<-0.1$, the microbe was inferred to have a topological niche in the control group. When a microbe had one positive and one negative values of $N i c h e_{i}^{\text {Indegree }}$ and $N i c h e_{i}^{\text {Betweenness }}$, it was considered to be part of an ambiguous topological niche between the control and bioaugmentation treatments.

\section{Inferring motifs from microbial networks}

Network motifs can reflect biological structures, connections, or regulations in a microbial network [34]. In this study, a network motif-detecting software called mfinder [35] was used to identify significant 3-and 4-node motifs at four interactive strengths-the 500, 1000, 1500, and 2000 strongest interactions-for two microbial networks. If a significant 4-node motif was repeatedly defined as having three or more than three interactive strengths, it was considered a representative 4-node motif in a microbial network. If a 4node motif was representative in the control or bioaugmented microbial networks, motif zscores calculated by mfinder were directly used to compare the significance of this motif in that microbial network.

\section{Assigning metabolic reactions for microbes}

Each microbial member was assigned metabolic functions by KEGG (Kyoto Encyclopedia of Genes and Genomes) reactions [36]. Queried items including "methane," "cellulose," "chitin," etc. were checked for 
every KEGG reactions among all microbial strains, which were retained if the genus or family of the strain also occurred in our abundance profiles.

\section{Results:}

\section{Creating an artificial subtle perturbation}

The so-called priority effect is the impact that the first colonized microbial population has in hampering the subsequent colonization of microbial immigrants and becoming a host defense mechanism that helps the human body confer colonization resistance against invading bacterial pathogens [22, 37]. Therefore, adding pregrown microbial cultures can disturb the microecological balance and re-establish the entire community as part of the pregrown culture's niche. One way to artificially create a microbial ecosystem under pressure from subtle perturbations is to add a bioaugmented microbial species to disrupt microbiota-mediated colonization resistance transitorily was currently proposed using an anaerobic digestion system. Bioaugmented microbial species were added to the mesophilic anaerobic digesters, and their disruptive effects on the anaerobic digestion system were evaluated once the biogas and methane production rose.

The supplemental microbial species added were selected based on a novel network-based approach coupled with four decisive criteria proposed by this study (Fig. 1). 1) Unlike commonly used bioaugmentation strategies based on well-known bacterial metabolic functions, key microbial families were identified using a comparative process based on topological differences between microbial networks from mesophilic and thermophilic anaerobic digestion systems [38]. Microbial families with topological niches and low levels of relative abundance in the mesophilic condition were marked as having the potential to enhance biogas or methane yields by bioaugmentation. 2) Checking the abundance of key microbial candidates at low levels is critical to reaching the goal of using bioaugmented species to amplify metabolic functions, resist the early colonizing community, and temporarily maximize the impact on a stable microecosystem. 3) Key microbial families should be aerobic in this study because they are easier to manipulate and cultivate. 4) Any bacterial species that could be independently isolated from anaerobic digesters in the laboratory and successfully cultivated in the medium could be retained and tested in the subsequent BMP and CSTR experiments, which were used to confirm that the subtle perturbation was successful by searching for increased biogas or methane yields. Microbial species that could cause a subtle perturbation in a stable microbial ecosystem were artificially identified following these four steps (Table S1).

\section{The artificial subtle perturbation}

To simulate an artificial subtle perturbation in a microbial ecosystem, two aerobic speciesElizabethkingia miricola (Family: Flavobacteriaceae) and Stenotrophomonas acidaminiphila (Family: Xanthomonadaceae) -with low abundance (Fig. S1) and topological differences [38] were isolated for use as bioaugmented factors to enhance biogas and methane production in mesophilic anaerobic 
digesters. Furthermore, Brachymonas denitrificans (Family: Comamonadaceae) and Escherichia coli (Family: Enterobacteriaceae) were selected for the negative control (Table S1).

A simplified, three-day version of the BMP test was used to quickly assess the effects of bioaugmentation using these four bacterial aerobes on biogas and methane production. Compared to the control group, $E$. miricola improved the mesophilic anaerobic digestion process of swine manure by the second day, and increased biogas and methane productions by $6.63 \pm 3.01 \%\left(P=2.8 \times 10^{-2}\right)$ and $29.07 \pm 8.00 \%(P=3 \times$ $\left.10^{-3}\right)$, respectively. $S$. acidaminiphila addition increased the biogas and methane production by $5.07 \pm 2.61 \%\left(P=4.2 \times 10^{-2}\right)$ and $14.37 \pm 3.99 \%\left(P=3 \times 10^{-3}\right)$, respectively, compared to the control group on the first day. Biogas and methane production increased due to an absolute increase in methane content $\left(\mathrm{CH}_{4}\right)$ of $1.97 \pm 0.30 \%$ (or $6.38 \pm 1.07 \%$ ) for S. acidaminiphila (or E. miricola) on the first (or second) day (Table S2). On the other hand, B. denitrificans and Escherichia coli were predicted to lower biogas and methane productions (Table S1), and the BMP test found that they did (Table S2).

To further appraise the efficacy of bioaugmented species, $S$. acidaminiphila was chosen because of how quickly it increased biogas and methane yields and was tested again in the CSTR reactors with the daily feeding of swine manure; this was carried out for longer than the BMP test and was regarded as a laboratory-scale biogas plant. The bioaugmented CSTR reactors using $S$. acidaminiphila showed a significantly enhanced GPR from $1.92 \pm 0.02$ to $2.05 \pm 0.02 \mathrm{~L} / \mathrm{L} /$ day $\left(P=8.13 \times 0^{-5}\right)$ and MPR from $1.24 \pm$ 0.02 to $1.32 \pm 0.03 \mathrm{~L} / \mathrm{L} /$ day $\left(P=1.72 \times 10^{-4}\right)$, meaning that it had successfully disrupted the stable microsystem (Fig. 2). To confirm the effect of $S$. acidaminiphila on biogas and methane yields, eight anaerobic digesters-four each for the control and bioaugmentation treatments-were used to reproduce the process (Fig. S2).

The final step to check whether the subtle disturbance was artificially made was to confirm the biodiversity of microbial communities from the mesophilic anaerobic digesters with and without $S$. acidaminiphila bioaugmentation. 16S rRNA sequencing information and taxonomic classification of time-series samples from anaerobic digesters with and without bioaugmentation were shown in Table S3 and S4. The similarities in the Shannon index and Chao-1 richness at the family level among all samples suggested that these biodiversity measurements failed to detect the subtle perturbation caused by the bioaugmentation (Table S5). Therefore, a subtle perturbation was successfully performed in the laboratory scale anaerobic digestion system based on the topological niche of microbial networks, meaning that this is a novel and prominent method for identifying potential influencers that can slightly disturb a stable microecosystem.

\section{The effects of subtle perturbation on microbial communities}

The next step was to identify the dynamic changes in microbial communities due to $S$. acidaminiphila perturbation that could not be detected by microbial biodiversity. Fourteen time-series samples from the mesophilic anaerobic digesters (CSTR) with and without the subtle perturbation were collected and 
manipulated by 16S rRNA amplicon sequencing analysis; after all the 16S rRNA sequence preprocessing steps were performed, 113 microbial families were identified with an average of 19,392 \pm 982 bacteria and archaea reads (Table S3 and S4).

Other than invariant microbial biodiversity, microbial communities also remained robust in their diversity species (Fig. 3(A)) with and without the addition of S. acidaminiphila in the mesophilic anaerobic digesters. Of these 101 highly overlapping taxa, 73.3\% were found showed a coherent presence in all samples; these were called the core microbial families, and they represented the main source of resistance to our slight impact manipulation using the bioaugmentation. In each condition, only 12 families were identified as being rare in relative abundance (Fig. 3(A)). When S. acidaminiphila was added, the microbial ecosystem preserved most of its members and only a small number of rare families emerged. This kind of conservation and stimulation in the microbial communities under pressure from subtle perturbation pushes the entire system in a discrepant situation in which the microbial diversities do not vary (Table S5) but do segregate strongly into two microbial populations-with and without bioaugmentation-based on principal component analysis (PCA) (Fig. 3(B)).

The clear separation between microbiomes indicated that the addition of S. acidaminiphila changed the microbial communities of anaerobic digesters. To clarify how microbial communities varied with operating time, daily feeding, and bioaugmentation, Bray-Curtis dissimilarity was used to measure the differences between pairs of abundance profiles (Fig. S3). There was more variation in the abundance of the bioaugmentation group $\left(6.8 \times 10^{-2}\right.$ to $\left.12.0 \times 10^{-2}\right)$ than the control one $\left(7.3 \times 10^{-2}\right.$ to $\left.9.7 \times 10^{-2}\right)$ (Fig. 3(C)). The variation among the pairwise control samples (blue in Fig. 3(C)) came from operational days, as the ingredients of the daily-feeding swine manure changed each day. The variation was larger among bioaugmented samples (green in Fig. 3(C)) because it was affected by the same daily feeding affect as well as the $S$. acidaminiphila. To eliminate the influence of daily feeding, Bray-Curtis dissimilarity between the control and bioaugmentation group on the same day was obtained, and it showed that the microbiome changed more and more owing to the added species (black in Fig. 3(C)).

To understand which microbes were influenced by bioaugmentation, three abundance featuresabundance level, differential abundance status, and correlated abundance patterns-were measured to determine their associations under bioaugmentation pressures. Thirty-three families with significantly differential abundances were identified as indicators of the weak impact of adding $S$. acidaminiphila on the entire microecosystem, 21 of which increased in abundance and 13 decreased (Fig. 4(A)). Of these 33 families, eight demonstrated an overall dominance: Lachnospiraceae (ID28), Porphyromonadaceae(ID1), Clostridiales_unclassified (ID59), Firmicutes_unclassified (ID69), Bacteria_unclassified (ID42), Bacteroidales_unclassified (ID43), Syntrophaceae(ID12), and Candidatus_Cloacamonas_unclassified (ID48). Apart from ID48 and ID12, these dominant microbes increased in abundance after bioaugmentation, but these changes did not appear to be correlated at all. Concerning the correlated abundance pattern, 12 highly correlated families were much less abundant (Fig. 4(A)). 
Therefore, a subtle perturbation could interfere with microbial communities across abundance levels, differential abundance statuses, and correlated abundance patterns (also called co-occurrence patterns). Here, we used a contingency table to decipher the intertwined relationship among these three abundance attributes. The separation of microbial families based on microbial abundance level was significantly associated with that by differential abundance $(P=0.00155)$ or correlated abundance pattern $(P=0.002)$, but microbes partitioned by abundance correlation were not significantly associated with those partitioned by differentially abundant attributes $(P=0.1654)$ (Fig. S4).

According to the bottom contingency table partitioned by abundance pattern and differential abundance status, microbial community could be divided into four parts. 1) Under the pressure from bioaugmentation, 13 families showed robust abundance profiles with similar abundance levels and unchanged (correlated) abundance patterns (Fig. 5(A) and Fig. S4); this might be the microbiome responsible for systematic colonization resistance. 2) Twelve families had correlated abundance patterns and differential abundance levels (yellow in Fig. 4(A) and red/green in Fig. 5(A)), and maintained the same abundance patterns through bioaugmentation; these might be the central families that helped achieve bioaugmentation. 3) Twenty-one families had different abundances but no correlated abundance patterns (not marked in Fig. 4(A)); they were largely influenced by the addition of S. acidaminiphila. 4) The final 55 families had similar abundance levels but no correlated abundance pattern; these appeared to react to the subtle perturbation randomly. However, more extensive research is necessary to make any definite claims about the function of these microbial communities.

The abundance profiles of $S$. acidaminiphila and methanogens were observed to examine the effects of the subtle perturbation on the entire microbiome. The invariant abundance level of Xanthomonadaceae (Fig. 4(B)), which S. acidaminiphila belongs to, suggested that anthropogenic microbial interference only disturbed the microecosystem at a small scale, but it could still temporarily improve biogas and methane yields (Fig. 2 and Fig. S2) after bioaugmentation. However, this change in biogas and methane levels during the anaerobic digestion process was not influenced by the prevalence of the archaeal community (Fig. 4(C)) or methanogens (Table 1), which are known to produce methane from substrates such as $\mathrm{H}_{2}$, acetate, methanol, and methylamine. The archaeal community contained methanogenic families, including Methanobacteriaceae, Methanospirillaceae, Methanomassiliicoccaceae, Methanomicrobiaceae, Methanosarcinaceae, and Methanotrichaceae, and four other unclassified families with a similar or lower abundance after $S$. acidaminiphila was added (Table 1). Therefore, the improvement in biogas and methane production after bioaugmentation may have been caused by bacterial communities rather than well-known archaeal methanogens. This is explored in the subsequent analysis. 
Table 1

Microbial abundance of archaeal families with and without bioaugmentation by adding S. acidaminiphila. Relative abundance from different time-series samples before and after bioaugmentation are represented as the average and standard error and were tested by Paired-samples Student's t test $\left(\mathrm{H}_{0}: \mathrm{U}_{\mathrm{C}}=\mathrm{U}_{\mathrm{B}}\right)$. Acetoclastic, hydrogenotrophic, and methylotrophic methanogens were defined by KEGG modules and are denoted $A$ (M00357: Acetate $\left.\diamond \mathrm{CH}_{4}\right), \mathrm{H}\left(\mathrm{M} 00567: \mathrm{CO}_{2} \diamond \mathrm{CH}_{4}\right), \mathrm{M} 1\left(\mathrm{M} 00356:\right.$ Methanol $\left.\diamond \mathrm{CH}_{4}\right)$, and M2 (M00563: Methylamine $\diamond \mathrm{CH}_{4}$ ).

\begin{tabular}{|c|c|c|c|}
\hline Archaeal families & C & B & P-value \\
\hline ID20: Methanotrichaceae (A) & $15.01 \pm 0.42$ & $15.41 \pm 0.63$ & 0.331 \\
\hline ID17:Methanosarcinaceae (A/H/M1/M2) & $0.03 \pm 0.01$ & $0.01 \pm 0.00$ & 0.088 \\
\hline ID21: Methanospirillaceae $(\mathrm{H})$ & $0.70 \pm 0.56$ & $0.56 \pm 0.06$ & $<0.001^{\star}$ \\
\hline ID13: Methanomicrobiaceae (H) & $0.15 \pm 0.02$ & $0.14 \pm 0.02$ & 0.328 \\
\hline ID18: Methanobacteriaceae (H/M1/M2) & $0.90 \pm 0.06$ & $0.59 \pm 0.03$ & $<0.001^{*}$ \\
\hline ID15: Methanomassiliicoccaceae(M1) & $0.14 \pm 0.02$ & $0.11 \pm 0.01$ & 0.076 \\
\hline ID39: Archaea_unclassified & $1.10 \pm 0.07$ & $1.05 \pm 0.05$ & 0.145 \\
\hline ID67: Euryarchaeota_unclassified & $0.68 \pm 0.04$ & $0.51 \pm 0.04$ & $0.014^{*}$ \\
\hline ID19: Methanomicrobiales_unclassified & $0.31 \pm 0.03$ & $0.34 \pm 0.02$ & 0.253 \\
\hline $\begin{array}{l}\text { ID95: Pacearchaeota_Incertae_Sedis } \\
\text { _AR13_unclassified }\end{array}$ & $0.32 \pm 0.08$ & $0.30 \pm 0.07$ & 0.246 \\
\hline
\end{tabular}

\section{Subtle perturbation and co-occurrence patterns}

Microbes with co-occurrence patterns were known to be driven by metabolic interactions and competition for resources [39], and had the potential to capture crucial community characteristics that might not be discovered in microbial diversity or abundance-based analysis [40, 41]. Based on 25 highly correlated microbial families $\left(\mathrm{r}_{\mathrm{C}_{\mathrm{i}} \mathrm{B}_{\mathrm{i}}}\right)$ with peculiar co-occurrence (ascent, descent, and convex) abundance patterns (Fig. 5(A) and S5), three microbial clusters (G1, G2, and G3) were established to capture different subsets of microbial families using $\mathrm{r}_{\mathrm{B}_{\mathrm{i}} \mathrm{B}_{\mathrm{j}}}$ and $\mathrm{r}_{\mathrm{C}_{i} \mathrm{C}_{j}}$ with and without bioaugmentation (Fig. 5(B) and S5). After $S$. acidaminiphila was added, the microbial families with $\mathrm{r}_{\mathrm{B}_{\mathrm{i}} \mathrm{B}_{\mathrm{j}}}$ captured by 25 microbes with co-occurrence $\left(\mathrm{r}_{\mathrm{C}_{\mathrm{i}} \mathrm{B}_{\mathrm{i}}}\right)$ patterns were different to those with $\mathrm{r}_{\mathrm{C}_{\mathrm{i}} \mathrm{C}_{\mathrm{j}}}$ without bioaugmentation. For the ascent abundance pattern, Porphyromonadaceae (ID1), Pseudomonadaceae(ID22) Peptococcaceae_2 (ID98), and Sunxiuqinia (ID113) were preserved in the $\mathrm{G} 1$ cluster, but were attached by different microbes with $\mathrm{r}_{\mathrm{C}_{\mathrm{i}} \mathrm{B}_{\mathrm{i}}}$ (Fig. 5(B) and S5). The G2 cluster contained microbes with a coherent descent abundance pattern, such as Corynebacteriaceae (ID14), Lachnospiraceae(ID28), Actinomycetaceae(ID34), Opitutaceae (ID93), Streptococcaceae (ID110), and Candidatus_Saccharibacteria_unclassified (ID51), of which only Lachnospiraceae showed a differential rise in abundance (Fig. 4). As the only member with a preserved convex abundance pattern in the G3 cluster, Xanthomonadaceae (ID109), the family of S. acidaminiphila, 
was linked by Clostridiaceae_1 (ID25) before bioaugmentation and by Prevotellaceae(ID29) after. Although Xanthomonadaceae was rarely associated with methane production in previous studies, Clostridiaceae_ 1 and Prevotellaceae are well-known bacterial families that are involved in hydrolysis, acidogenesis, acetogenesis, and methanogenesis. Furthermore, $S$. acidaminiphila seemed to act as a driver that gathered more core microbes to have co-occurrence abundance patterns and more cooccurrence links after bioaugmentation in the G1 and G3 clusters (Fig. S5); this might be an innate mechanism to retain special regulations or enhance the production of biogas and methane in the microbial ecosystem.

\section{The methanogenic microbiota among co-occurrence clusters}

Six methanogens changed in abundance (Table 1), and those with similar (ID13, 15, 17, and 20) or lower (ID18 and 21) abundance levels after $S$. acidaminiphila was added suggest the existence of unknown bacteria that improved biogas or methane yields. A systematic way to screen bacterial members that might generate methane could rely on metabolic reactions from the KEGG database [42]. The KEGG database found that bacteria containing the KEGG reactions R10204 and R09339 are believed to produce methane by metabolizing methylphosphonate [43] and methylmercury [44] with carbon-phosphorus lyase (EC 4.7.1.1) and alkylmercury lyase (EC 4.99.1.2).

Some bacteria use methylphosphonate as a source of phosphorus-an essential element for nucleic acids, carbohydrates, and phospholipids-when there is a lack of phosphate; this includes most gramnegative bacteria [42, 43] and species in Enterobacteriaceae (ID16), Pseudomonadaceae (ID22), Coriobacteriaceae (ID23), Desulfomicrobiaceae(ID63), Desulfuromonadaceae (ID64), Geobacteraceae (ID72), and Rhodobacteraceae (ID103) and those distributed in three co-occurrence clusters (Table S6). Other than Coriobacteriaceae (ID23), which had low abundance, most of these families were relatively rare. Two rare families, Desulfomicrobiaceae (ID63) and Desulfuromonadaceae (ID64), increased dramatically in abundance after bioaugmentation. Desulfomicrobiaceae (ID63) (Fig. 3(A)), for example, initially could not be measured, but it became detectable after bioaugmentation, suggesting that the addition of S. acidaminiphila stimulated Desulfomicrobiaceae growth.

Methylmercury is formed from inorganic mercury directly by microbes in certain aquatic environments or indirectly by acute anthropogenic poisoning, e.g. industrial discharge and large scale combustion of fossil fuels or wastes containing inorganic mercury $[44,45]$. Methylmercury bioaccumulates in aquatic food chains, making fish meal fed to growing pigs the main source of methylmercury from anaerobic digesters. Five families-Corynebacteriaceae(ID14), Dietziaceae(ID65), Geobacteraceae (ID72), Moraxellaceae (ID89), and Sphingomonadaceae (ID108)-possess the enzyme alkylmercury lyase (Table S6). The abundance of these families remained low (Fig. S5). Dietziaceae (ID65) was the only one that increased differentially in abundance in the G1 co-occurrence cluster after bioaugmentation (Fig. 4 and Table S6). These microbial families with the KEGG reactions R10204 and R09339 were connected by seven central correlated microbes: Enterococcaceae (ID5), Acidaminococcaceae(ID10), 
Enterobacteriaceae(ID16), Fibrobacteraceae(ID68), Clostridiales_Incertae_Sedis_XI (ID56), Syntrophorhabdus (ID86), and Methanomicrobiales_unclassified (ID19). Therefore, co-occurrence clusters were used to form a list of microbes that may be involved in methane production, and this is further discussed in the discussion section.

Furthermore, KEGG reactions also suggested that Xanthomonadaceae (ID109) have other functions, including the degradation of cellulose (R11307) and chitin (R01206 and R02334). There reactions contained cellulase (EC 3.2.1.4) and chitinase (EC 3.2.1.14) to degrade common feed ingredients for swine. Although the abundance level of Xanthomonadaceae (ID109) was similar with and without bioaugmentation, 28 microbial families (data not shown) that could degrade cellulose and chitin were collected in this study and showed significantly different increases in abundances $(P=0.01)$ under bioaugmentation pressure. Therefore, adding $S$. acidaminiphila transitorily raised the biogas and methane production, possibly due to the enhancement of bacterial methanogenesis and improvement of carbohydrate degradation. This study points to new possibilities for future microbiome research.

\section{Subtle perturbation and microbial network}

There were 25 microbial families with highly correlated abundance patterns under the bioaugmentation condition but not the control one, and these guided the microsystem into three co-occurrence clusters (Fig. 5) and turned more core families together in a co-occurrence manner (Fig. S5). These microbial families with correlated abundance patterns with and without bioaugmentation may play a role in regulating the entire microbiome and reach the goal of improving biogas and methane production. To decipher the regulatory roles of these 25 microbes with co-occurrence patterns, microbial networks, network topologies, and network motifs from the control and bioaugmentation were used to discern any regulatory patterns in the microbial ecosystem (Fig. 6).

First, two microbial networks with and without bioaugmentation were constructed from time-series samples. The largest microbial network was composed of 12,656 interactions and 113 microbes. Then, the difference between the microbial networks was measured in a systematic way using two topological indices, betweenness, and indegree centrality under different levels of interactive strengths. Microbial families that had higher betweenness and indegree centrality with (or without) bioaugmentation are shown in the yellow (or red) boxes (Fig. 6(A)). For the negative control, 12 families specific to conditions with or without bioaugmentation (Fig. 3) were assembled into networks and their definite topological niche was shown (Fig. 6(A)). Although microbial families with high abundance levels, abundance differential statuses, and abundance correlated patterns were frequently considered as critical for comparison, they were not always defined as important in an intertwined microsystem (Fig. 6(A)). For example, two correlated families, Clostridiaceae_1 (ID25) and Comamonadaceae (ID60), had higher abundance levels after bioaugmentation, but showed no topological difference after $S$. acidaminiphila was added (Fig. 6(A)). However, Methanomicrobiaceae (ID 13) had no specific abundance properties, but showed topological niches under the pressure of bioaugmentation. 
Then, 3-node and 4-node network motifs were analyzed for each microbial network to determine the regulatory patterns from microbial networks. The strongest microbial interactions (top 500 to 2000) were selected to obtain significant 3-node motif types (Table S7). For these 3-node motifs, M3-36 and M3-78 were specific to the microbial network with bioaugmentation. M3-36 was a convergence pattern of microbial relations and M3-78 possessed the bidirectional patterns, convergence and divergence, for microbial interactions. The added species stimulated communication behaviors between microbes. The microsystem could induce or repress a microbial member more quickly with M3-36, and the inferences of an essential microbial family could enlarge signals instantly using M3-78. In addition, M3-74 and M3-98 were important motifs for both microbial networks with and without bioaugmentation. M3-74 was a cascade motif that triggered sequential repressions or activations [46]. The M3-98 motif was a feedback loop that occurs when outputs of a system amplifies or inhibits the system to generate sustained oscillations for biological rhythms [47].

To understand the significance and conservation of 3-node motifs in microbial networks, significant 4node motifs (Table S8) were further identified to determine whether or not these 3-node motifs occurred reoccurred. Five 4-node motifs-M4-404, M4-406, M4-908, M4-4682, and M4-5004-were simultaneously defined as significant among microbial networks before and after bioaugmentation (green region in Table S8). All three 3-node motifs-M3-36, M3-74, and M3-78-were portions of these 4-node motifs (not feedback motif M3-98). For example, M3-74 was a part of M4-404. The frequencies of these five consistent 4-node motifs in the two microbial networks are listed in Table S9 and visualized in Figure S6. Adding species induced stronger microbial interactions conveying a long chain communication by M4406 and M4-908, and weaker interactions conveying additional support by a short chain cascade (M4404 and M4-4682). However, without the help of additive species, the strong interactions were contributed by a mixture of long chain (M4-5004 and M4-406) and 3-node cascade (M4-404 and M4-4682) motif niches, and weaker interactions only preserved the long chain motifs (Fig. S6). The comparison of niche flow among the 4-node motifs revealed potential benefits of adding species, as the niche motifs under different interactive strength levels from M4-406 (top 500), M4-908 (top 1000) to M4-908, M4-404 and M4-4682 (top 2000) formed a hierarchical regulatory structure to function in the ecosystem in a parallel way.

Then, seven and fourteen 4-node motifs specific to microbial networks with and without bioaugmentation were determined, respectively (Table S8). Under the condition of adding S. acidaminiphila, a quite different group of significant 4-node motifs, especially looped motifs (orange regions in Table S8), were identified and seemingly featured in the M3-98 feedback motif. For theses 4-node motifs with feedback loops, higher frequencies of central correlated microbial families $\left(\mathrm{r}_{\mathrm{C}_{\mathrm{i}} \mathrm{B}_{\mathrm{i}}}\right)$ (Fig. $5(\mathrm{~A})$ ) were observed after bioaugmentation (Fig. S7). When the 500 strongest interactions were selected (Fig. 5(A)), seven out of ten 4-node looped motifs had a higher proportion of M3-98 feedback motifs with correlated microbes with bioaugmentation (Fig. S7(A)). For instance, $29.27 \%$ of the M4-4418 motif contained the M3-98 feedback motif with correlated microbes (Fig. S7(A)) when the top 500 strong interactions were kept. Similarly, the microbial network without bioaugmentation produced many 4-node loop motifs, such as M4-6874 and M4-13150, which were also contributed to by microbes with central correlated abundance patterns (Fig. 
S7(A)). When the 2000 strongest interactions were used (Fig. S7(B)), a similar association between cooccurrence microbes and the M3-98 feedback loop among 4-node motifs was found. Six 4-node motifsM4-330, M4-4418, M4-4998, M4-5062, M4-6870, and M4-13142-had more correlated 3-node M3-98 feedback patterns after adding S. acidaminiphila when the 500 and 2000 strongest interactions were used (Fig. S7). M4-6874 still showed a higher percentage of the correlated M3-98 motif without bioaugmentation.

Three abundance features and co-occurrence clustering types were matched with principal component loadings to provide a substantial link between abundance attributes and principal component loadings and clearly decipher the potential drivers separating microbial communities (Fig. 3(B)). The main force driving the first and second principal component was derived from discriminating G1 and G2 cooccurrence clusters and four differentially abundant categories, respectively (Fig. 6(B)). These findings led us to understand the importance of recognizing co-occurrence abundance patterns in microbiome research (Fig. 6(B)), as they might play critical regulatory roles in microbial ecosystems.

To determine the metabolic functional changes between two microbial networks with and without the additional species, two small-scale microbial networks-including families with well-known methanerelated metabolic pathways, hydrolysis, acidogenesis, acetogenesis, methanogenesis, and desulfurization, based on our previous study [38]-were constructed (Fig. 6(C) and (D)) to examine changes in the topological niche among the five methane-related pathways under bioaugmentation pressure. More interactions between Porphyromonadaceae (ID1) and Bacteroidaceae (ID2) increased hydrolysis. This was consistent with the observation that adding $S$. acidaminiphila increased chitin and cellulose degradation. Apart from Veillonellaceae(ID7), all microbes that could undergo acidogenesis contributed equally to microbial networks (Fig. 6(A)). Then, Syntrophaceae (ID12) and Acidaminococcaceae (ID10) showed a topological niche after adding S. acidaminiphila, but another two were more important without bioaugmentation in the process of acetogenesis. Methanogens did not show increased abundance (Table 1), but all except for Methanomicrobiaceae (ID13) showed a slight topological niche. Only one syntrophic methanogen, Corynebacteriaceae (ID14), showed a significant interactive topology (Fig. 6(A)). The ambiguous topological niche of Synergistaceae (ID30) revealed a larger betweenness centrality after the subtle perturbation and a larger indegree centrality without any perturbation (Fig. 6(A) and (D)). The metabolic improvement by multifunctional Clostridiaceae_1 (25) and Lachnospiraceae (28) might generate stronger impacts than that by Prevotellaceae (ID29).

These results indicated that integrating intertwined relationships among microbes into a microbial ecosystem helps detect and decipher changes in the microbiome in response to a mild disturbance. Cooccurrence abundance patterns are a common way to investigate the microbial interactions. In this study, the regulatory role of correlated microbial families (Fig. 5) was further established. Microbes kept special abundance patterns to fine-tune the major metabolic functions and modified the interactive relationships to form hierarchical niche motifs that could respond to the perturbation in a more sensitive way. Therefore, we provided a systematic decomposition of the entire microbial ecosystem after a subtle disturbance by adding S. acidaminiphila at a very different aspect.

Page $17 / 33$ 


\section{Discussion:}

Microbial diversity is a common bioindicator of ecosystem functions [48], but it is not sensitive enough to detect chronic and subtle environmental perturbations [5, 9]. Gradual environmental changes will delay evolutionary adaptations in eukaryotes [49], and the gradual exposure of RNA viruses like Ebola, SARS, and influenza to new host species will make them more dangerous because of the major genetic changes in these pathogens [50]. Detecting or monitoring gradual changes are important for preventing global changes, ecological disturbances, and human-induced pollution from worsening. Instead of biodiversity, non-random patterns in microbial species co-occurrence and associated metrics are being integrated to amplify the differences that subtle perturbation make [20,51], e.g. a temperature increase of $1{ }^{\circ} \mathrm{C}$ for 5 years [19], soil contamination with mercury for several decades [16], and annual litter decomposition [17]. Our analysis provides a framework for studying microbial communities, co-occurrence, and networks under mild anthropogenic perturbation by adding $S$. acidaminiphila.

In this study, a network based approach was first proposed to design and measure a microbial ecosystem after a subtle perturbation. Based on network topologies, predicted key species were added to a mesophilic anaerobic digestion microsystem to slightly interfere with the priority effect from the species that arrived first in the communities, also called colonization resistance [22,50]. The addition of $S$. acidaminiphila yielded increased biogas and methane production; this represents the transitory disturbance to the microbial ecology, and the invariant microbial species represent a protective mechanism in this microsystem that prevents the colonization and overgrowth of new bacterial species. Therefore, this study takes a step toward designing a novel laboratory-scale that differentiates microbial topological niches in response to subtle perturbation pressure. This whole new way of artificially disturbing a microbial ecosystem at a small scale provides a chance for researchers to observe and investigate changes in microbial communities in response to gradual and subtle perturbation. In addition, the generalization of such laboratory-scale experimental findings to real environmental changes might help monitor and sound the alarm about mild changes from chronic atmospheric pollution [19], agricultural practices [17], and metallic contaminants in the soil [16] at an early stage, which can then be countered with ecosystem management strategies.

Although most taxa were maintained after $S$. acidaminiphila was added, changes in abundance level, differential abundance status, correlated abundance pattern, network motif, and microbial network were detected. For three abundance attributes, differential abundance status and correlated abundance did not associate with each other, but each was significantly associated with abundance level. The conventional way to decipher microbial communities with high abundance levels is not capable of adequately explaining intertwined microbial relationships. However, co-occurrence patterns and network topology results underscore the importance of recognizing regulatory interactive behaviors between microbes. In three co-occurrence clusters, seven central correlated microbes-Enterococcaceae(ID5), Acidaminococcaceae (ID10), Enterobacteriaceae(ID16), Fibrobacteraceae(ID68), Clostridiales_Incertae_Sedis_XI(ID56), Syntrophorhabdus (ID86), and Methanomicrobiales_unclassified (ID19)-were linked with microbes containing the KEGG reactions R10204 and R09339. 
Syntrophorhabdus aromaticivorans (family Syntrophorhabdus (ID86)) was the first cultured anaerobe found to be responsible for degrading phenol into acetate and methane in a syntrophic relationship with hydrogenotrophic methanogens [52]. The methane generating potential of Syntrophorhabdus (ID86) was ignored in KEGG reactions; however, the co-occurrence patterns between Syntrophorhabdus (ID86) and microbes with R10204 and R09339-Pseudomonadaceae(ID22), Desulfuromonadaceae (ID64), and Dietziaceae (ID65)-addressed the underlying importance of generating methane, which was proven in a literature survey [52]. Furthermore, Syntrophorhabdus (ID86) increased in abundance with bioaugmentation, increased in abundance with bioaugmentation, but not consistently across all samples. Syntrophorhabdus (ID86) coupled with Methanomicrobiales_unclassified (ID19), Acidaminococcaceae (ID10), and Clostridiales_Incertae_Sedis_XI (ID56) were also shown to have critical roles in the network topology after $S$. acidaminiphila was added, whereas Methanomicrobiales_unclassified (ID19) might include uncultured hydrogenotrophic methanogens that are syntrophic with Syntrophorhabdus (ID86). Acetogenesis of Acidaminococcaceae (ID10) could definitely raise the efficacy of biogas and methane yields. Besides, some gram-negative short rods in Escherichia and Enterobacter [53] (family Enterobacteriaceae (ID16)) could enlarge the formation of methylmercury and indirectly improve methane production, even in the presence of oxygen. Concerning the daily feeding of swine manure to the mesophilic anaerobic digesters, it was inevitable that some oxygen entered the microsystem, thus allowing for some bacterial methanogenesis.

These improvements mentioned above might be triggered by adding $S$. acidaminiphila, an aerobe that was first isolated from a lab-scale upflow anaerobic sludge blanket reactor treating petrochemical wastewater [54]. S. acidaminiphila has multiple carbon sources, including acetate, crotonate, fumarate, DL-Lactate, pyruvate, and succinate. Furthermore, it can degrade N-Acetylglucosamine [54]-the monomeric unit of the polymer chitin and a major component of the cell walls of most fungi and bacteria -and be resistant to various antimicrobial agents [55]. Consistent with the potential metabolic functions of the family of Xanthomonadaceae based on KEGG reactions, Xanthomonadaceae conveyed the ability to degrade cellulose (R11307) and chitin (R01206 and R02334). The degradation of N-Acetylglucosamine implies that $S$. acidaminiphila in anaerobic digesters increase fiber digestion and the ability to break down or reuse dead microbes.

Bacteria-oriented methanogenesis and carbon degradation explain why biogas and methane yields increased in a low-abundance methanogenic population after $S$. acidaminiphila was added. In addition, the microbial networks suggest a transition from methanogens that consume a combination of acetate, hydrogen, and methanol (Methanotrichaceae, Methanospirillaceae, and Methanobacteriaceae) to purely hydrogenotrophic types (Methanomicrobiaceae and Methanomicrobiales_unclassified). Although the concentration of organic acids could not be detected in this study, it is possible that the added $S$. acidaminiphila rapidly exhausted most of the acetate at anaerobic digesters and created a hydrogenabundant environment for hydrogenotrophic methanogens. However, more rigorous and extensive laboratory experiments are necessary to support this proposition and make any definitive claims along these lines. 
Co-occurrence and model-based microbial networks are two popular approaches based on different rationales to decipher dynamic microbial ecosystems [14,32,56-58]. The biggest difference between the two prediction strategies is whether the interaction direction can be inferred. Commonly used methods that use co-occurrence networks to infer microbial interactions are based on non-directional association measurements, such as Pearson and Spearman correlation [16, 18, 59], Bray-Curtis distance [60], or covariance estimation [61]. Model-based networks can follow certain regression models, such as Bayesian statistics, Lotka-Volterra, and a variety of sparse regressions [32, 62-64]. However, this is the first study to combine co-occurrence and model-based microbial networks (e.g. Lotka-Volterra), and it indicates the regulatory roles that co-occurrence microbes might play in a dynamic microbial network. Although the addition of $S$. acidaminiphila to anaerobic digesters did not directly interfere with microbial community diversities, it did change the microbial ecosystem by enhancing network motifs and motivating bacteria-mediated methanogenesis by feedback loop and cascade signaling motifs.

Consistent with previous studies $[65,66]$, feedback loop regulation had a great impact on cell growth and microbial biofuel production, where the toxic effects of biofuels for cell growth could be mitigated by expressing efflux pumps to export biofuels from the microbes. Furthermore, the overall performance of biofuel production depended on a cascade process, including the efficient pretreatment of influent sludge, more short-chain fatty acids, and higher conductivities in the fermentative liquid [67]. In our study, the stronger microbial interactions highlighted long chain regulatory cascade motifs, M4-406, and parallelly the weaker interactions featured shorter chain cascade motifs, M4-404 and M4-4682, which may have boosted the microbial cascade process by switching to different interactive strengths after bioaugmentation. Our study demonstrates for the first time under mildly perturbed environments that the purpose of hierarchical regulatory motifs launched by co-occurrence members might form a functional module to respond to the dynamic surroundings instantly.

\section{Conclusions:}

Previous efforts to characterize ecological fitness and adaptation have primarily been conducted based on the response of microbial diversities to some disturbance. In the coming years, dynamic microbial ecological studies will increasingly be applied to detect subtle environmental perturbations. We present a systematic approach for handling time-series microbial communities to detect slight changes in microbial abundance between two populations with and without subtle perturbation. This method can be generalized to dynamic experiments in a wide variety of fields and provides a predictive direction and landscape for further research and experimental designs. In order to obtain more reliable and objective support, future research should search for evidence of these regulatory network motifs under a microbial ecological process and use them to decipher intertwined relations among microbes.

\section{List Of Abbreviations:}

CSTR: continuous stirred tank reactors 
HRT: hydraulic retention time

TS: total solids

BMP: biochemical methane potential

COD: chemical oxygen demand

GCN: 16S rRNA gene copy number

GAP: generalized association plots

KEGG: Kyoto Encyclopedia of Genes and Genomes

PCA: principal component analysis

\section{Declarations}

\section{Ethics approval and consent to participate}

Not applicable

\section{Consent for publication}

Not applicable

\section{Availability of data and material}

All data generated or analysed during this study are included in this published article and its supplementary information files.

\section{Competing interests}

The authors declare that they have no competing interests.

\section{Funding}

This work was supported by the Ministry of Science and Technology of Taiwan [Grant No: MOST 1082321-B-002-024], and Sustainability Science Research Project, Academia Sinica. 


\section{Authors' contributions}

GTWS performed the statistical and network analysis for the dynamic microbial abundance dataset, and wrote the manuscript. CYC (corresponding author), DW, GTWS and ACL conceived the project. ACL and YCC operated the anaerobic digesters. CYW, CYC, and FCHW processed the samples for amplicon sequencing. CYW isolated the pure culture from effluent samples. All authors read and approved the final manuscript.

\section{Acknowledgements}

Thanks to Noah Last of Third Draft Editing for his English language editing.

\section{References}

1. Cardinale BJ, Duffy JE, Gonzalez A, Hooper DU, Perrings C, Venail P, Narwani A, Mace GM, Tilman D, Wardle DA, et al: Biodiversity loss and its impact on humanity. Nature 2012, 486:59-67.

2. Loreau M, Naeem S, Inchausti P, Bengtsson J, Grime JP, Hector A, Hooper DU, Huston MA, Raffaelli D, Schmid B, et al: Biodiversity and ecosystem functioning: current knowledge and future challenges. Science 2001, 294:804-808.

3. Brown JH, Whitham TG, Morgan Ernest SK, Gehring CA: Complex species interactions and the dynamics of ecological systems: long-term experiments. Science 2001, 293:643-650.

4. Willis KJ, Bhagwat SA: Ecology. Biodiversity and climate change. Science 2009, 326:806-807.

5. Pradhan A, Bhaumik P, Das S, Mishra M, Khanam S, Hoque BA, Mukherjee I, Thakur AR, Chaudhuri SR: Phytoplankton diversity as indicator of water quality for fish cultivation. 2008.

6. Uttah EC, Uttah C, Akpa P, Ikpeme E, Ogbeche J, Usip L, Asor J: Bio-survey of plankton as indicators of water quality for recreational activities in Calabar River, Nigeria. Journal of Applied Sciences and Environmental Management 2008, 12.

7. Arora H: Rotifera as indicators of trophic nature of environments. Hydrobiologia 1966, 27:146-159.

8. Grizzle RE: Pollution indicator species of macrobenthos in a coastal lagoon. Marine ecology progress series Oldendorf 1984, 18:191-200.

9. Paerl HW, Dyble J, Moisander PH, Noble RT, Piehler MF, Pinckney JL, Steppe TF, Twomey L, Valdes LM: Microbial indicators of aquatic ecosystem change: current applications to eutrophication studies. FEMS Microbiology Ecology 2003, 46:233-246.

10. Paerl HW, Dyble J, Moisander PH, Noble RT, Piehler MF, Pinckney JL, Steppe TF, Twomey L, Valdes LM: Microbial indicators of aquatic ecosystem change: current applications to eutrophication studies. FEMS Microbiol Ecol 2003, 46:233-246.

11. Spellerberg IF, Fedor PJ: A tribute to Claude Shannon (1916-2001) and a plea for more rigorous use of species richness, species diversity and the 'Shannon-Wiener'Index. Global ecology and 
biogeography 2003, 12:177-179.

12. Simpson EH: Measurement of diversity. Nature 1949, 163:688.

13. Chao A, Chiu $\mathrm{CH}$, Jost L: Phylogenetic diversity measures based on Hill numbers. Philos Trans $R$ Soc Lond B Biol Sci 2010, 365:3599-3609.

14. Weiss S, Van Treuren W, Lozupone C, Faust K, Friedman J, Deng Y, Xia LC, Xu ZZ, Ursell L, Alm EJ, et al: Correlation detection strategies in microbial data sets vary widely in sensitivity and precision. ISME J 2016, 10:1669-1681.

15. Zelezniak A, Andrejev S, Ponomarova O, Mende DR, Bork P, Patil KR: Metabolic dependencies drive species co-occurrence in diverse microbial communities. Proceedings of the National Academy of Sciences 2015, 112:6449-6454.

16. Zappelini C, Karimi B, Foulon J, Lacercat-Didier L, Maillard F, Valot B, Blaudez D, Cazaux D, Gilbert D, Yergeau E: Diversity and complexity of microbial communities from a chlor-alkali tailings dump. Soil Biology and Biochemistry 2015, 90:101-110.

17. Sauvadet M, Chauvat M, Cluzeau D, Maron P-A, Villenave C, Bertrand I: The dynamics of soil microfood web structure and functions vary according to litter quality. Soil Biology and Biochemistry 2016, 95:262-274.

18. Zhou J, Deng Y, Luo F, He Z, Yang Y: Phylogenetic molecular ecological network of soil microbial communities in response to elevated CO2. MBio 2011, 2:e00122-00111.

19. Karimi B, Meyer C, Gilbert D, Bernard N: Air pollution below WHO levels decreases by $40 \%$ the links of terrestrial microbial networks. Environmental Chemistry Letters 2016, 14:467-475.

20. Karimi B, Maron PA, Chemidlin-Prevost Boure N, Bernard N, Gilbert D, Ranjard L: Microbial diversity and ecological networks as indicators of environmental quality. Environmental Chemistry Letters 2017, 15:265-281.

21. Sprockett D, Fukami T, Relman DA: Role of priority effects in the early-life assembly of the gut microbiota. Nat Rev Gastroenterol Hepatol 2018, 15:197-205.

22. Grainger TN, Letten AD, Gilbert B, Fukami T: Applying modern coexistence theory to priority effects. Proc Natl Acad Sci U S A 2019, 116:6205-6210.

23. Liu A-C, Chou $C-Y$, Chen $L-L$, Kuo $C-H$ : Bacterial community dynamics in a swine wastewater anaerobic reactor revealed by 16 S rDNA sequence analysis. Journal of biotechnology 2015, 194:124131.

24. Owen W, Stuckey D, Healy Jr J, Young L, McCarty P: Bioassay for monitoring biochemical methane potential and anaerobic toxicity. Water research 1979, 13:485-492.

25. Yu Y, Lee C, Kim J, Hwang S: Group-specific primer and probe sets to detect methanogenic communities using quantitative real-time polymerase chain reaction. Biotechnology and bioengineering 2005, 89:670-679.

26. Sundberg C, Al-Soud WA, Larsson M, Alm E, Yekta SS, Svensson BH, Sorensen SJ, Karlsson A: 454 pyrosequencing analyses of bacterial and archaeal richness in 21 full-scale biogas digesters. FEMS 
Microbiol Ecol 2013, 85:612-626.

27. Magoc T, Salzberg SL: FLASH: fast length adjustment of short reads to improve genome assemblies. Bioinformatics 2011, 27:2957-2963.

28. Schloss PD, Westcott SL, Ryabin T, Hall JR, Hartmann M, Hollister EB, Lesniewski RA, Oakley BB, Parks DH, Robinson CJ, et al: Introducing mothur: open-source, platform-independent, communitysupported software for describing and comparing microbial communities. Appl Environ Microbiol 2009, 75:7537-7541.

29. Edgar RC: UPARSE: highly accurate OTU sequences from microbial amplicon reads. Nat Methods 2013, 10:996-998.

30. Angly FE, Dennis PG, Skarshewski A, Vanwonterghem I, Hugenholtz P, Tyson GW: CopyRighter: a rapid tool for improving the accuracy of microbial community profiles through lineage-specific gene copy number correction. Microbiome 2014, 2.

31. Wu H-M, Tien Y-J, Chen C-h: GAP: A graphical environment for matrix visualization and cluster analysis. Computational Statistics \& Data Analysis 2010, 54:767-778.

32. Shaw GT, Pao YY, Wang D: MetaMIS: a metagenomic microbial interaction simulator based on microbial community profiles. BMC Bioinformatics 2016, 17:488.

33. Bastian M, Heymann S, Jacomy M: Gephi: an open source software for exploring and manipulating networks. ICWSM 2009, 8:361-362.

34. Milo R, Shen-Orr S, Itzkovitz S, Kashtan N, Chklovskii D, Alon U: Network motifs: simple building blocks of complex networks. Science 2002, 298:824-827.

35. Kashtan N, Itzkovitz S, Milo R, Alon U: Efficient sampling algorithm for estimating subgraph concentrations and detecting network motifs. Bioinformatics 2004, 20:1746-1758.

36. Ogata H, Goto S, Sato K, Fujibuchi W, Bono H, Kanehisa M: KEGG: Kyoto Encyclopedia of Genes and Genomes. Nucleic Acids Res 1999, 27:29-34.

37. Litvak Y, Baumler AJ: The founder hypothesis: A basis for microbiota resistance, diversity in taxa carriage, and colonization resistance against pathogens. PLoS Pathog 2019, 15:e1007563.

38. Shaw GT, Liu AC, Weng CY, Chou CY, Wang D: Inferring microbial interactions in thermophilic and mesophilic anaerobic digestion of hog waste. PLoS One 2017, 12:e0181395.

39. Levy R, Borenstein E: Metabolic modeling of species interaction in the human microbiome elucidates community-level assembly rules. Proceedings of the National Academy of Sciences 2013, 110:12804-12809.

40. Read T, Lamothe L, Le Boulch M, Cauquil L, Gabinaud B, Bannelier C, Balmisse E, Destombes N, Bouchez O, Gidenne T: Diversity and co-occurrence pattern analysis of cecal microbiota establishment at the onset of solid feeding in young rabbits. Frontiers in microbiology 2019, 10:973.

41. McGregor K, Labbe A, Greenwood CM: MDiNE: A model to estimate differential co-occurrence networks in microbiome studies. bioRxiv 2019:544122. 
42. Muto A, Kotera M, Tokimatsu T, Nakagawa Z, Goto S, Kanehisa M: Modular architecture of metabolic pathways revealed by conserved sequences of reactions. J Chem Inf Model 2013, 53:613-622.

43. Jochimsen B, Lolle S, McSorley FR, Nabi M, Stougaard J, Zechel DL, Hove-Jensen B: Five phosphonate operon gene products as components of a multi-subunit complex of the carbonphosphorus lyase pathway. Proceedings of the National Academy of Sciences 2011, 108:1139311398.

44. Marvin-DiPasquale M, Agee J, McGowan C, Oremland RS, Thomas M, Krabbenhoft D, Gilmour CC: Methyl-mercury degradation pathways: a comparison among three mercury-impacted ecosystems. Environmental Science \& Technology 2000, 34:4908-4916.

45. Compeau G, Bartha R: Sulfate-reducing bacteria: principal methylators of mercury in anoxic estuarine sediment. Appl Environ Microbiol 1985, 50:498-502.

46. Tran NT, Mohan S, Xu Z, Huang CH: Current innovations and future challenges of network motif detection. Brief Bioinform 2015, 16:497-525.

47. Tsai TY, Choi YS, Ma W, Pomerening JR, Tang C, Ferrell JE, Jr.: Robust, tunable biological oscillations from interlinked positive and negative feedback loops. Science 2008, 321:126-129.

48. Naeem S, Thompson LJ, Lawler SP, Lawton JH, Woodfin RM: Declining biodiversity can alter the performance of ecosystems. Nature 1994, 368:734-737.

49. Gorter FA, Aarts MM, Zwaan BJ, de Visser JA: Dynamics of Adaptation in Experimental Yeast Populations Exposed to Gradual and Abrupt Change in Heavy Metal Concentration. Am Nat 2016, 187:110-119.

50. Morley VJ, Turner PE: Dynamics of molecular evolution in RNA virus populations depend on sudden versus gradual environmental change. Evolution 2017, 71:872-883.

51. Tulloch AIT, Chades I, Lindenmayer DB: Species co-occurrence analysis predicts management outcomes for multiple threats. Nat Ecol Evol 2018, 2:465-474.

52. Qiu YL, Hanada S, Ohashi A, Harada H, Kamagata Y, Sekiguchi Y: Syntrophorhabdus aromaticivorans gen. nov., sp. nov., the first cultured anaerobe capable of degrading phenol to acetate in obligate syntrophic associations with a hydrogenotrophic methanogen. Appl Environ Microbiol 2008, 74:2051-2058.

53. Hamdy MK, Noyes OR: Formation of methyl mercury by bacteria. App/ Microbiol 1975, 30:424-432.

54. Assih EA, Ouattara AS, Thierry S, Cayol J-L, Labat M, Macarie H: Stenotrophomonas acidaminiphila sp. nov., a strictly aerobic bacterium isolated from an upflow anaerobic sludge blanket (UASB) reactor. International journal of systematic and evolutionary microbiology 2002, 52:559-568.

55. Huang Y-T, Chen J-M, Ho B-C, Wu Z-Y, Kuo RC, Liu P-Y: Genome sequencing and comparative analysis of Stenotrophomonas acidaminiphila reveal evolutionary insights into sulfamethoxazole resistance. Frontiers in microbiology 2018, 9:1013.

56. Berry D, Widder S: Deciphering microbial interactions and detecting keystone species with cooccurrence networks. Frontiers in microbiology 2014, 5:219. 
57. Tulloch Al, Chadès I, Lindenmayer DB: Species co-occurrence analysis predicts management outcomes for multiple threats. Nature ecology \& evolution 2018, 2:465-474.

58. Xiao Y, Angulo MT, Friedman J, Waldor MK, Weiss ST, Liu YY: Mapping the ecological networks of microbial communities. Nat Commun 2017, 8:2042.

59. Faust K, Sathirapongsasuti JF, Izard J, Segata N, Gevers D, Raes J, Huttenhower C: Microbial cooccurrence relationships in the human microbiome. PLoS Comput Biol 2012, 8:e1002606.

60. Faust K, Raes J: Microbial interactions: from networks to models. Nature Reviews Microbiology 2012, 10:538-550.

61. Kurtz ZD, Müller CL, Miraldi ER, Littman DR, Blaser MJ, Bonneau RA: Sparse and compositionally robust inference of microbial ecological networks. PLOS Comput Bio/ 2015, 11.

62. Bucci V, Tzen B, Li N, Simmons M, Tanoue T, Bogart E, Deng L, Yeliseyev V, Delaney ML, Liu Q: MDSINE: Microbial Dynamical Systems INference Engine for microbiome time-series analyses. Genome biology 2016, 17:121.

63. Stein RR, Bucci V, Toussaint NC, Buffie CG, Rätsch G, Pamer EG, Sander C, Xavier JB: Ecological modeling from time-series inference: insight into dynamics and stability of intestinal microbiota. PLoS Comput Biol 2013, 9.

64. Noyes N, Cho K-C, Ravel J, Forney LJ, Abdo Z: Associations between sexual habits, menstrual hygiene practices, demographics and the vaginal microbiome as revealed by Bayesian network analysis. PLoS One 2018, 13.

65. Dunlop MJ, Keasling JD, Mukhopadhyay A: A model for improving microbial biofuel production using a synthetic feedback loop. Systems and synthetic biology 2010, 4:95-104.

66. Harrison M, Dunlop M: Synthetic feedback loop model for increasing microbial biofuel production using a biosensor. Frontiers in microbiology 2012, 3:360.

67. Liu W, He Z, Yang C, Zhou A, Guo Z, Liang B, Varrone C, Wang A-J: Microbial network for waste activated sludge cascade utilization in an integrated system of microbial electrolysis and anaerobic fermentation. Biotechnology for biofuels 2016, 9:83.

\section{Figures}


Thermophilic AnD

Temp: $55^{\circ} \mathrm{C}$

TS: $5 \%$

HRT: 15 days
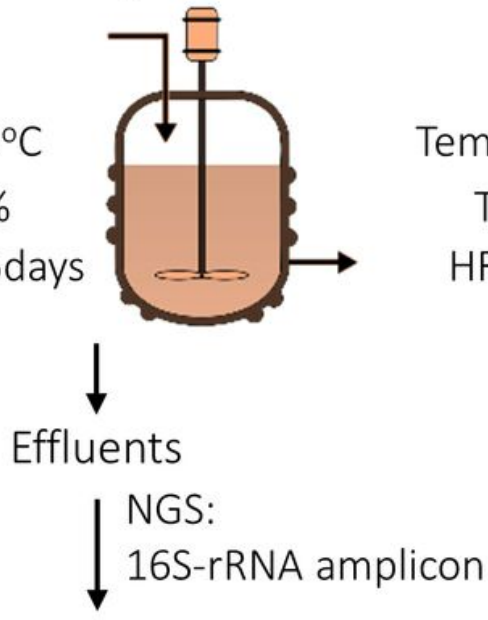

Times-series microbial abundance table

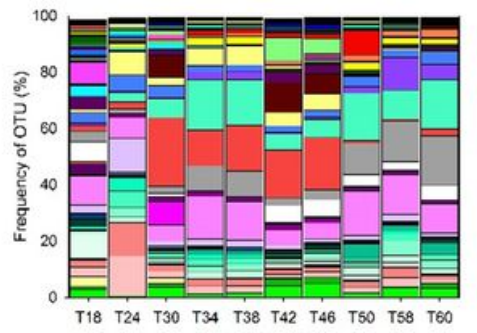

Time points (day)
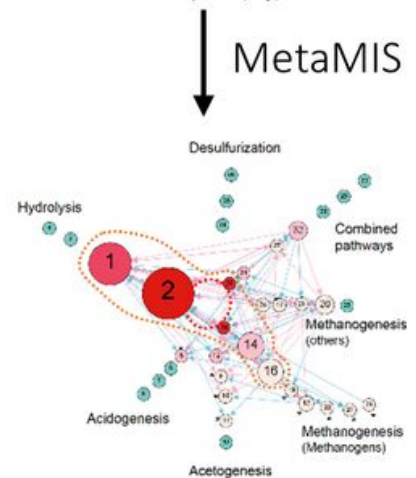

Acetogenesis

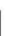

Temp: $37^{\circ} \mathrm{C}$

TS: $5 \%$

HRT: 15 days

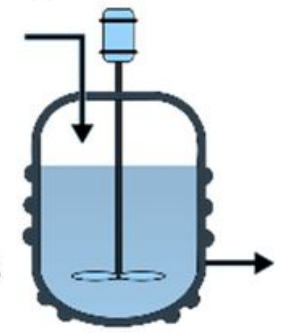

Effluents

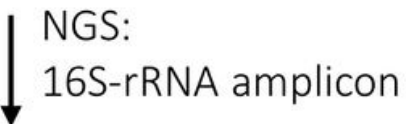

Times-series microbial abundance table

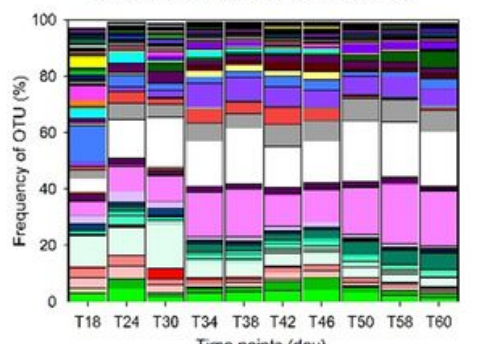

Time points (day)

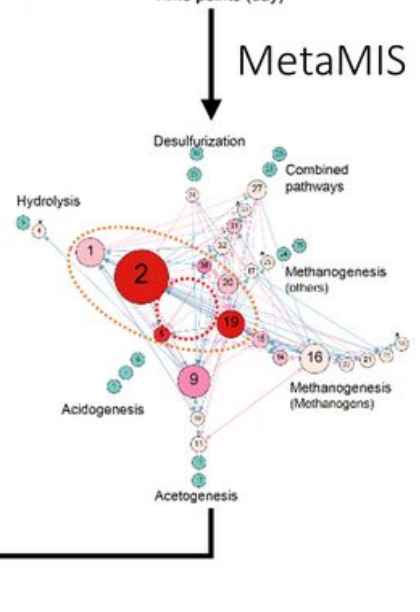

Microbes with topological niche
Isolate pure culture

under aerobic condition

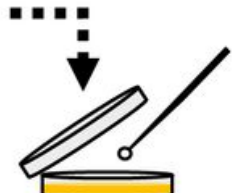

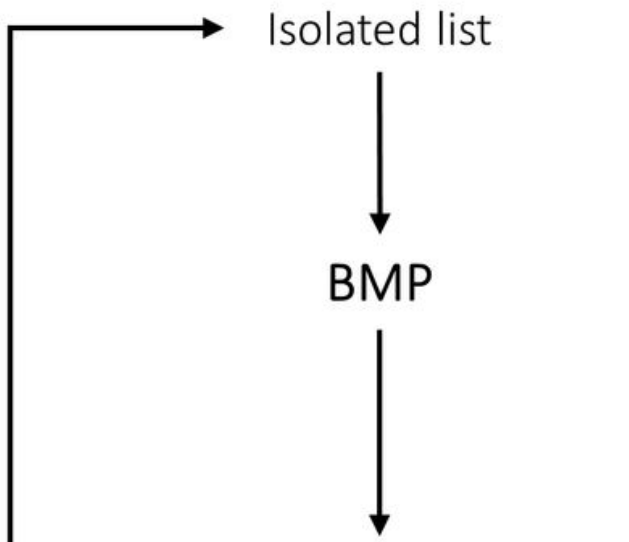

CSTR

\section{Figure 1}

A flowchart of the network-based bioaugmentation approach. The mesophilic and thermophilic microbial networks were derived from a previous study [38]. 

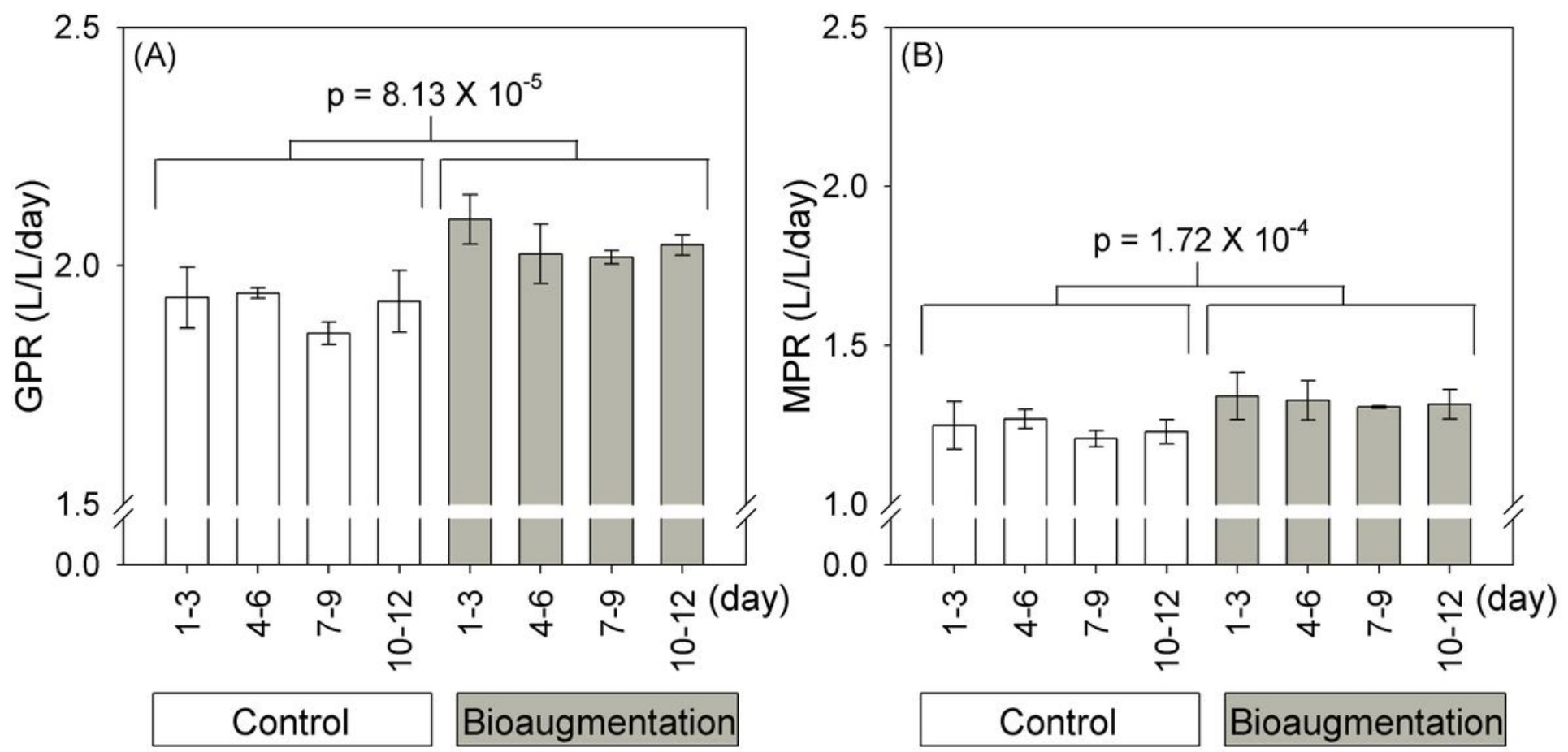

\section{Figure 2}

The effect of adding S. acidaminiphila to daily-fed CSTR. Compared to the control group, adding S. acidaminiphila significantly raised the level of (A) GPR and (B) MPR. Two-sample Student's t test was used to compare the differences in GPR or MPR between the control and bioaugmentation treatments. 
(A)

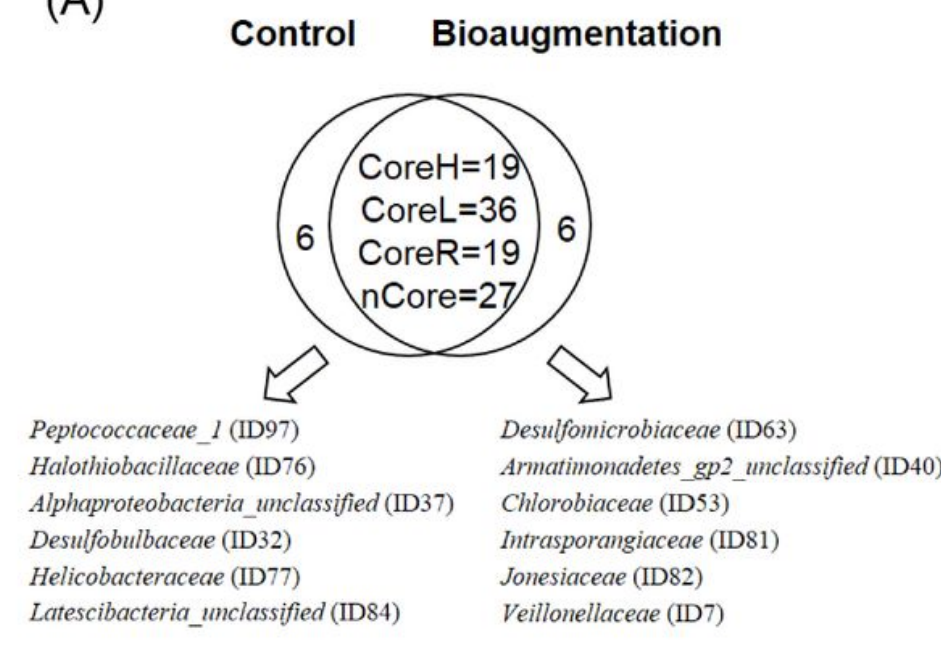

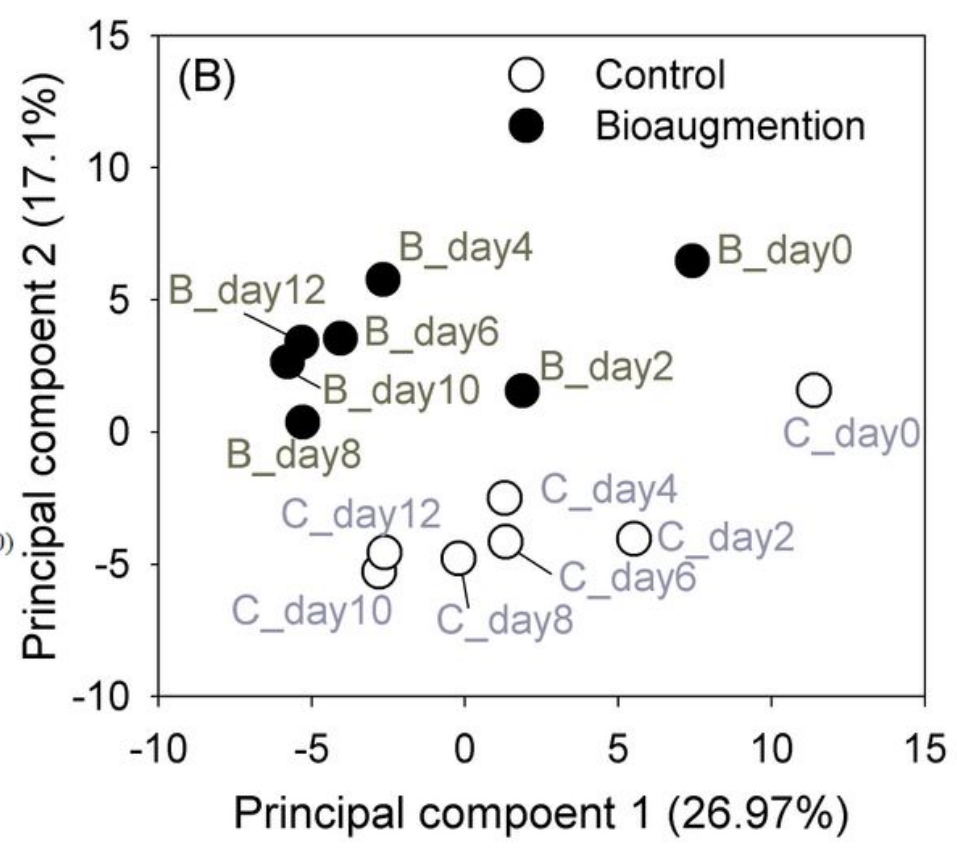

(C)

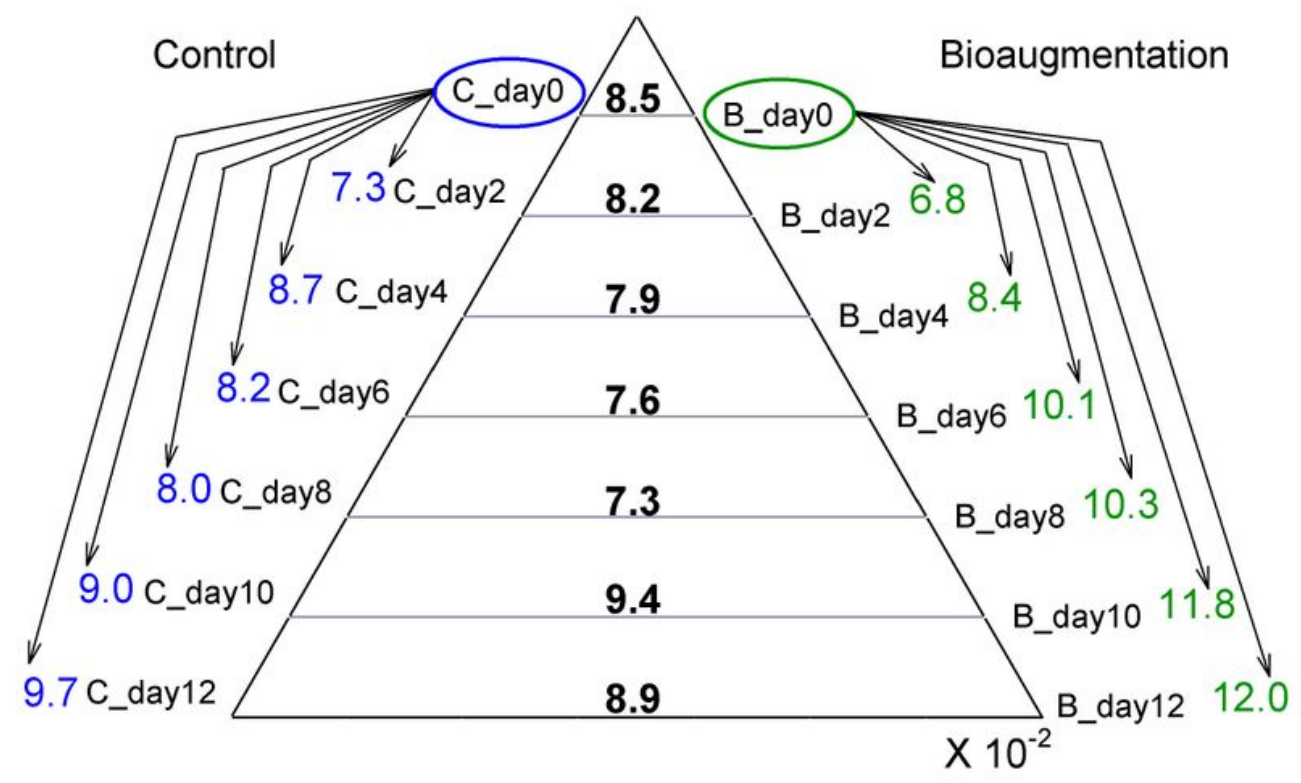

\section{Figure 3}

The effects of bioaugmentation on microbial communities. (A) Venn diagram of sequenced microbes with and without bioaugmentation. (B) Principal component analysis clearly separated samples, with $44.07 \%$ of variance explained by the first two components. (C) Bray-Curtis dissimilarities for pairwise time-series samples with and without bioaugmentation. The blue and green values represent Bray-Curtis dissimilarities between the $\mathrm{C}_{-}$day 0 and B_day 0 sample and all other control and bioaugmentation samples, respectively. The black values represent the dissimilarities between the bioaugmentation and control samples for each operational day, e.g. C_day 0 and B_day0 conferred a Bray-Curtis dissimilarity of 8.5×10-2. 

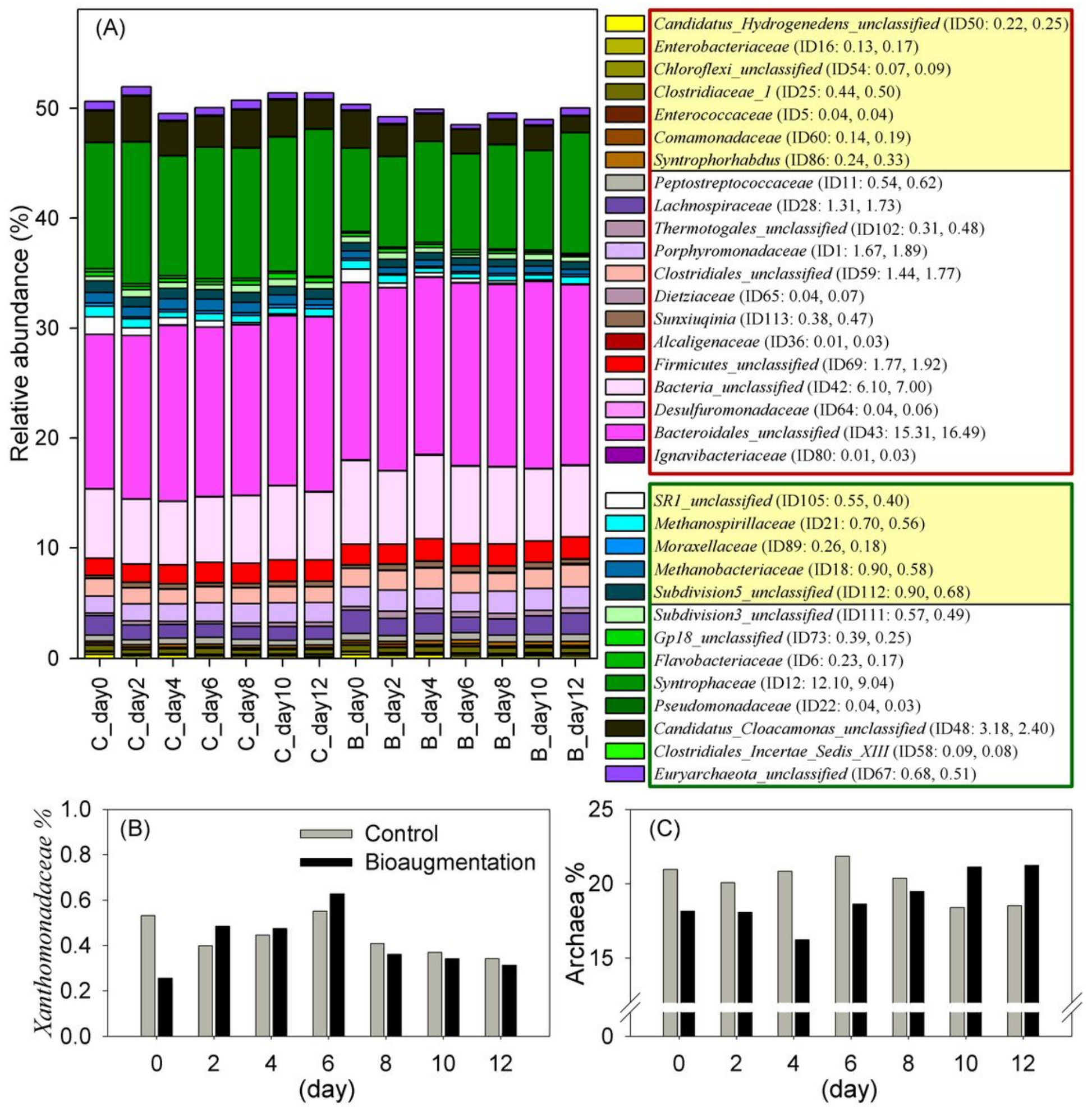

\section{Figure 4}

The influence of bioaugmentation on microbial communities. The abundance profiles of (A) 33 differential abundant families, (B) family Xanthomonadaceae (C:0.44 $\pm 0.03, \mathrm{~B}: 0.41 \pm 0.05 ; \mathrm{P}=0.576$ ), and (C) the archaeal population ( $\mathrm{C}: 20.14 \pm 0.48, \mathrm{~B}: 18.99 \pm 0.67 ; \mathrm{P}=0.332)$. The microbial population is denoted as mean and standard error. Paired-samples Student's t test (H0: UC=UB) for population comparison. 


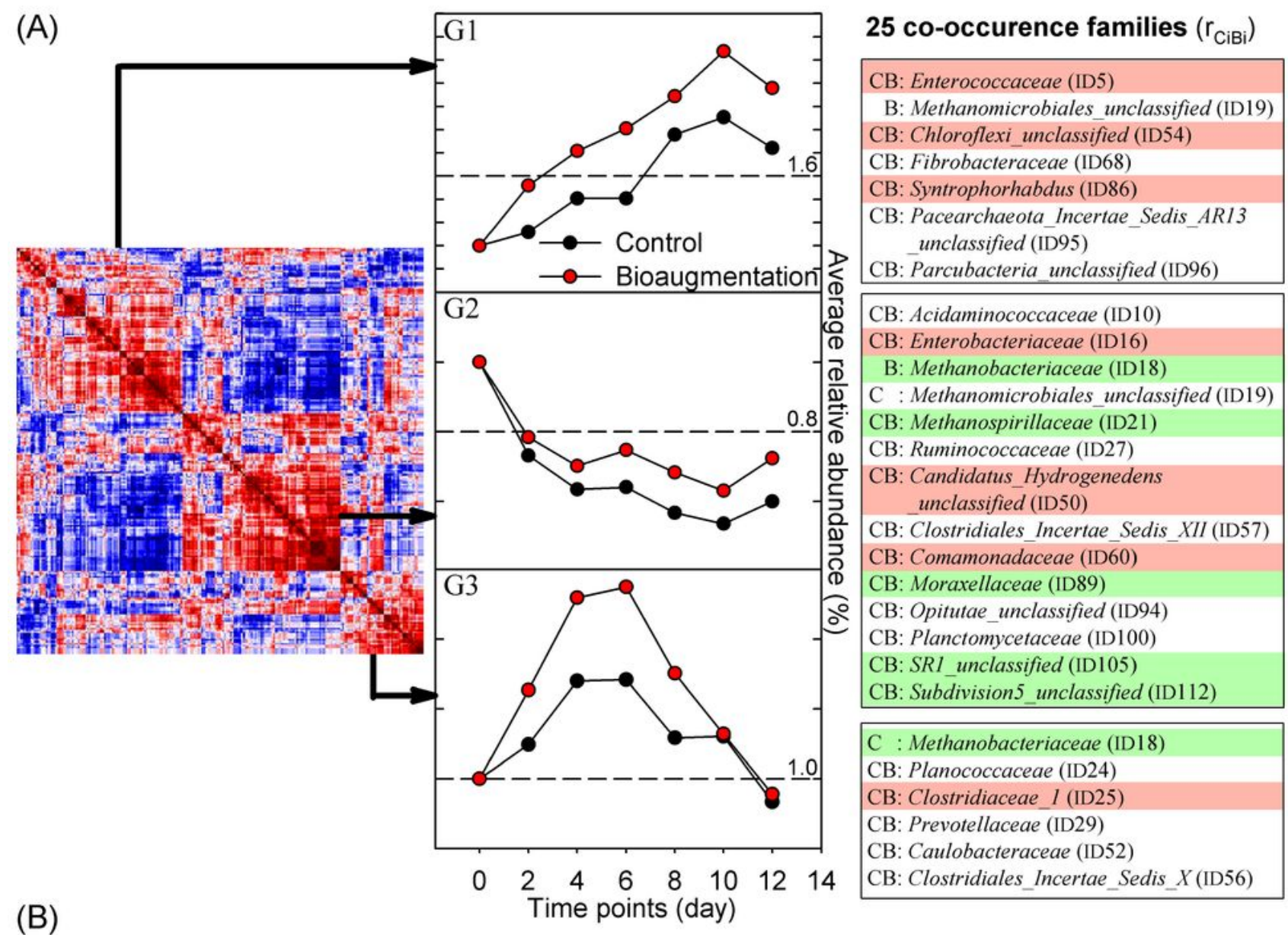

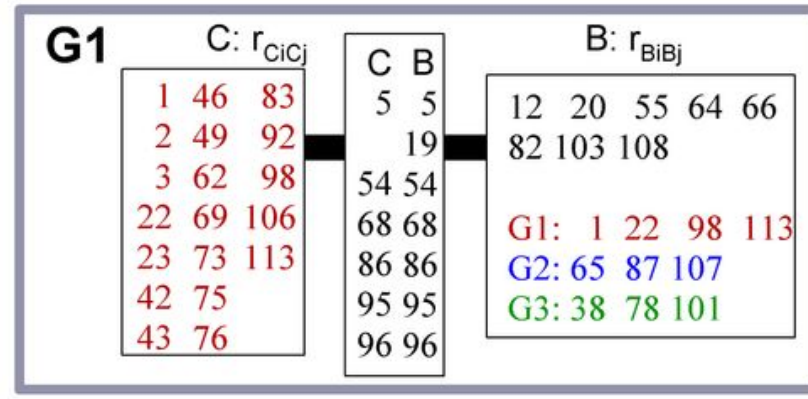

\section{G3}

\begin{tabular}{|c|c|c|}
\hline C: $r_{\mathrm{CiCj}}$ & C & $B: r_{B i B j}$ \\
\hline $11 \quad 101$ & 18 & G1: $23 \quad 46 \quad 69$ \\
\hline $17 \quad 109$ & 2424 & G2: 693171 \\
\hline $\begin{array}{l}37 \\
38\end{array}$ & $\begin{array}{lll}25 & 25 \\
20 & 20\end{array}$ & G3:109 \\
\hline 39 & 5252 & \\
\hline 78 & 5656 & \\
\hline
\end{tabular}

\begin{tabular}{|c|c|c|c|}
\hline G2 & $\mathrm{C}: \mathrm{r}_{\mathrm{CiCj}}$ & $C D$ & $B: r_{B i B j}$ \\
\hline & 661 & $\begin{array}{cc}10 & 10\end{array}$ & $\begin{array}{lllll}8 & 41 & 44 & 48 & 63\end{array}$ \\
\hline & 965 & 1616 & $\begin{array}{llll}81 & 85 & 88 & 104\end{array}$ \\
\hline & 1370 & $\begin{array}{lll}10 & 10 \\
18 & -25\end{array}$ & \\
\hline & 1471 & 19 & G1: $42 \quad 49 \quad 73$ \\
\hline & 1572 & 2121 & G2: $14 \quad 28 \quad 34 \quad 51$ \\
\hline & 2874 & $27 \begin{array}{ll}1 & 27\end{array}$ & 93110 \\
\hline & 3177 & $\begin{array}{ll}1 \\
50 & 50\end{array}$ & G3: $11 \quad 17$ \\
\hline & 3284 & 5757 & \\
\hline & 3387 & 6060 & \\
\hline & 3493 & $\begin{array}{ll}89 & 89\end{array}$ & \\
\hline & 51107 & 9494 & \\
\hline & 58110 & 100100 & \\
\hline & 59 & 105105 & \\
\hline & & 112112 & \\
\hline
\end{tabular}

\section{Figure 5}

Co-occurrence clusters revealed different co-occurrence abundance patterns. (A) 25 microbial families with highly correlated abundance patterns before (control) and after bioaugmentation. (B) Three cooccurrence clusters determined using the families in (A). Microbes with different rises and declines in abundance from Figure $4(A)$ are in red and green. $r_{-}\left(C_{-} i\right.$ B_ $i$ ) captured conserved abundance patterns 
before and after bioaugmentation. Based on the central correlated microbes $r_{-}\left(C_{-} i\right.$ B_i $\left.i\right)$, microbes with similar abundance pattern, $r_{-}\left(C_{-} i C_{-} j\right)$ or $r_{-}\left(B_{-} i B_{-} j\right)$, were identified under control or bioaugmentation.

(A)

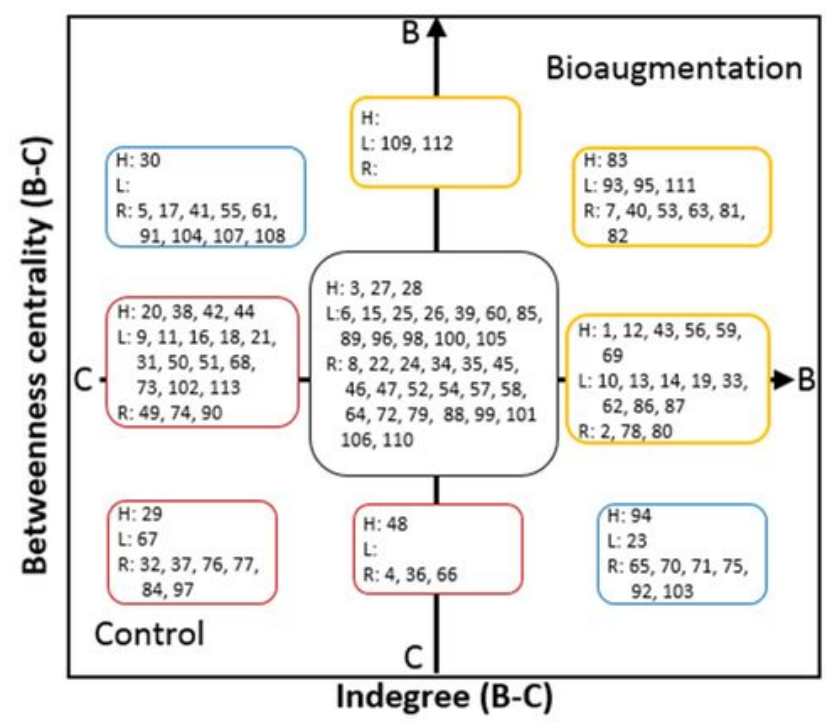

(C)

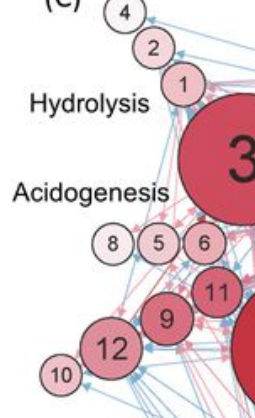

Acetogenesis
Desulfurization

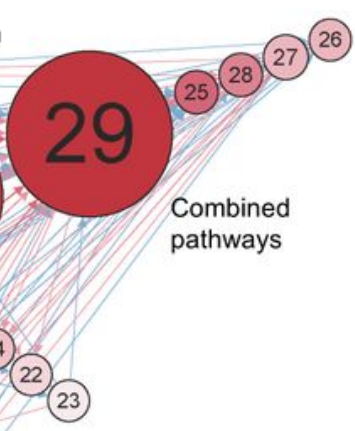

Methanogenesis (others)

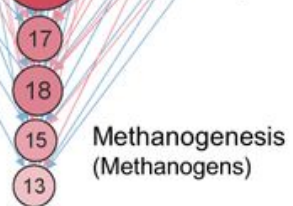

(B)
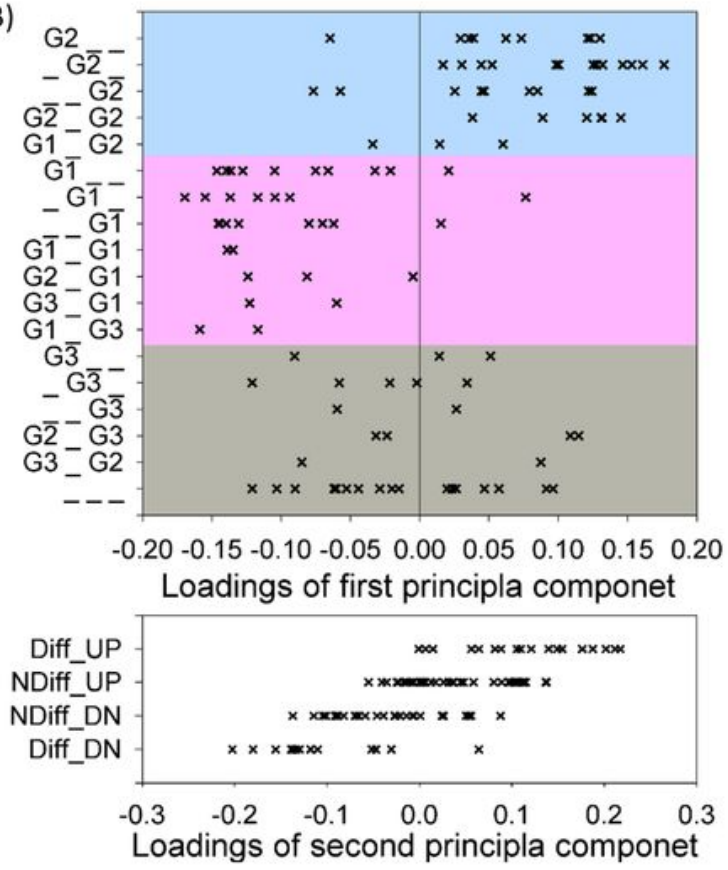

(D)

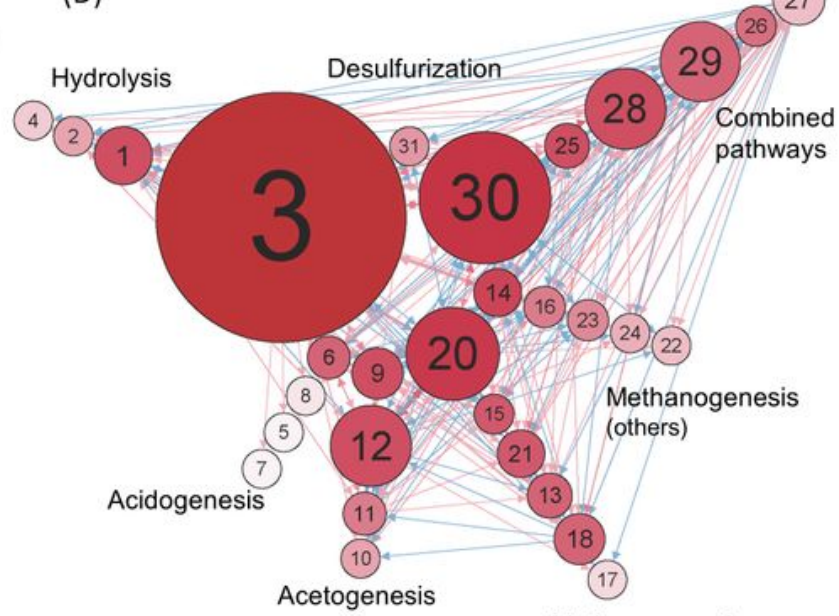

Methanogenesis (Methanogens)

\begin{tabular}{|c|c|c|c|c|c|c|c|}
\hline \multicolumn{2}{|r|}{ Hydrolysis } & Acetogenesis & \multicolumn{2}{|r|}{ Methanogenesis: Methanogens } & \multicolumn{2}{|r|}{ Methanogenesis: others } & Combined pathways \\
\hline 1 & p1: Porphyromonadaceae & \begin{tabular}{|l|l|}
9 & p3: Erysipelotrichaceae \\
\end{tabular} & 13 & p4: Methanomicrobiaceae & 14 & 4: Corynebacteriaceae & \begin{tabular}{|l|l}
25 & $p 1,2:$ Clostridiaceae 1 \\
\end{tabular} \\
\hline 2 & p1: Bacteroidaceae & 10 $p 3$ : Acidaminococcaceae & 17 & p4: Methanosarcinaceae & 16 & p4: Enterobacteriaceae & \begin{tabular}{|l|l|}
26 & $p 2,3$ : Lactobacillaceae \\
\end{tabular} \\
\hline 3 & p1: Spirochaetaceae & \begin{tabular}{|l|l|}
11 & p3: Peptostreptococcaceae \\
\end{tabular} & 18 & p4: Methanobacteriaceae & 22 & 4: Pseudomonadaceae & \begin{tabular}{|l|l|}
27 & p1-4: Ruminococcaceae \\
\end{tabular} \\
\hline 4 & p1: Marinilabiaceae & \begin{tabular}{|l|l|}
12 & p3: Syntrophaceae \\
\end{tabular} & 20 & p4: Methanotrichaceae & 23 & p4: Coriobacteriaceae & 28 p1-4:Lachnospiraceae \\
\hline \multicolumn{2}{|r|}{ Acidogenesis } & & & p4: Methanospirillaceae & 24 & p4: Planococcaceae & \begin{tabular}{|l|l}
29 & p1-4:Prevotellaceae \\
\end{tabular} \\
\hline 5 & p2: Enterococcaceae & & & \multirow[t]{4}{*}{ p4: Methanomassiliicoccaceae } & & & 30 p1-4:Synergistaceae \\
\hline 6 & p2: Flavobacteriaceae & & & & & & \begin{tabular}{|c|} 
Desulfurization \\
\end{tabular} \\
\hline 7 & p2: Veillonellaceae & & & & & & \multirow[t]{2}{*}{\begin{tabular}{|l|l|}
31 & deS: Syntrophobacteraceae \\
\end{tabular}} \\
\hline 8 & p2: Aerococcaceae & & & & & & \\
\hline
\end{tabular}

\section{Figure 6}

Microbial networks were helpful for detecting the differences with and without adding S. acidaminiphila. (A) A systematic visualization comparing two microbial networks based on betweenness and indegree centrality in the control and bioaugmentation groups. (B) Co-occurrence clusters (G, G2, or G3) and 
differential abundance status explained the variance in the first and second principal components. Principal component loadings were weights of microbial families on the first (or second) principal component. Two small-scale microbial networks including microbes matched to well-known functions are displayed (C) without and (D) with the addition of S. acidaminiphila. Diff_UP or Diff_DN indicate microbes with differential abundance that rose or declined, respectively (Fig. 4). NDiff_UP or NDiff_DN indicate microbes with non-differential abundances that rose or declined after adding species.

\section{Supplementary Files}

This is a list of supplementary files associated with this preprint. Click to download.

- Additionalfile.pdf 